\title{
Kinematic Steering Law for Conically-Constrained Torque-Limited Spacecraft Attitude Control
}

by

\author{
M. F. Diaz Ramos \\ Electronics Engineer, Universidad de Buenos Aires, Argentina, 2012
}

\author{
A thesis submitted to the \\ Faculty of the Graduate School of the \\ University of Colorado in partial fulfillment \\ of the requirements for the degree of \\ Master of Science
}

Ann and H. J. Smead Department of Aerospace Engineering Sciences 
This thesis entitled:

Kinematic Steering Law for Conically-Constrained Torque-Limited Spacecraft Attitude Control written by M. F. Diaz Ramos

has been approved for the Ann and H. J. Smead Department of Aerospace Engineering Sciences

Hanspeter Schaub

Jay McMahon

Daniel Kubitschek

Date

The final copy of this thesis has been examined by the signatories, and we find that both the content and the form meet acceptable presentation standards of scholarly work in the above mentioned discipline. 
Diaz Ramos, M. F. (M.S., Ann and H. J. Smead Department of Aerospace Engineering Sciences) Kinematic Steering Law for Conically-Constrained Torque-Limited Spacecraft Attitude Control Thesis directed by Hanspeter Schaub

Orientation constrained attitude control is a requirement in most spacecraft missions. A novel technique for attitude control of a spacecraft subjected to an arbitrary number of constraints in the form of inclusion and exclusion conic regions using a kinematic steering law and a rate-based attitude servo system is presented. The control laws are rate and torque bounded. The tracking errors are defined using Modified Rodrigues Parameters to yield a non-singular description. Lyapunov theory and logarithmic barrier potential functions are used to derive a kinematic steering law suitable for both attitude regulation and tracking scenarios. Conditions for constraint enforcement under limited-control-torque capability are studied. Numerical examples of regulation and tracking problems are shown. A Monte Carlo simulation is performed to illustrate control enforcement under bounded-torque control. 


\section{Dedication}

To Camilo, Meli, Flor, Agus, my parents, and friends. 


\section{Acknowledgements}

I would like to thank Dr. Hanspeter Schaub for all his support during the process, not only academic, but also emotional. Additional thanks go to committee members, Dr. Jay McMahon and Dr. Daniel Kubitschek. Finally, I would love to thank my labmates and friends at the Autonomous Systems Laboratory who have been so supportive in many different ways. 


\section{Contents}

\section{Chapter}

$\begin{array}{lll}1 & \text { Introduction } & 1\end{array}$

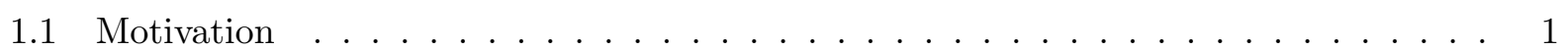

1.2 Existing Constrained Attitude Control Frameworks . . . . . . . . . . . . . . . . . . 3

1.3 Thesis Research Goals $\ldots \ldots \ldots \ldots \ldots$

2 Unconstrained Steering Law Using Modified Rodrigues Parameters 10

2.1 Modified Rodrigues Parameters . . . . . . . . . . . . . . . . . . . . . . 10

2.2 Rigid-Body Dynamics with Reaction Wheels $\ldots \ldots \ldots$. . . . . . . . . . . . . 12

2.3 Unconstrained MRP Steering Law . . . . . . . . . . . . . . . . . . . . . . . 13

$2.3 .1 \quad$ MRP Steering Law $\ldots \ldots \ldots \ldots \ldots \ldots \ldots$

$2.3 .2 \quad$ Servo Sub-System $\ldots \ldots \ldots \ldots \ldots \ldots \ldots$

$2.3 .3 \quad$ A Word on Numerical Computation $\ldots \ldots \ldots \ldots$

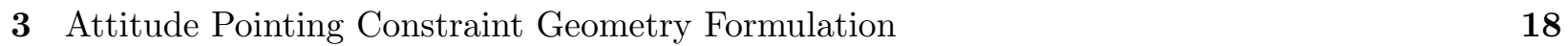

3.1 Constraint Classification $\ldots \ldots \ldots \ldots \ldots \ldots \ldots$

3.2 Conic Constraints $\ldots \ldots \ldots \ldots \ldots$

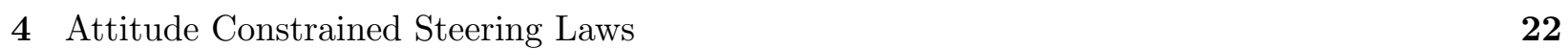

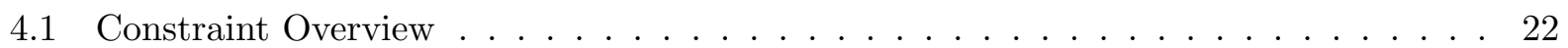

$4.2 \quad$ Attitude Constrained Regulation Steering Law . . . . . . . . . . . . . . . . . . 23 
$4.3 \quad$ Attitude Constrained Tracking Steering Law . . . . . . . . . . . . . . . . . . . . . 25

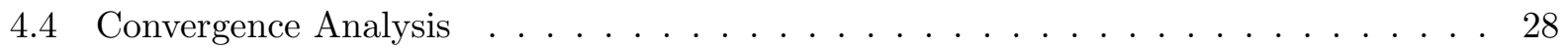

4.5 Numerical Simulations $\ldots \ldots \ldots \ldots$. . . . . . . . . . . . . . . . . . 30

4.5 .1 Slew Problem . . . . . . . . . . . . . . . . . . . . . . . . . . . . 31

4.5.2 Saddle-Points in the Regulation Problem . . . . . . . . . . . . . . . . 31

4.5 .3 Tracking Problem $\ldots \ldots \ldots \ldots \ldots \ldots$

\begin{tabular}{|lll}
\hline $\mathbf{5}$ & The Effect of Bounded Control Torque & $\mathbf{3 7}$
\end{tabular}

5.1 Constraint avoidance with finite control torque $\ldots \ldots \ldots \ldots$. . . . . . . . 37

$5.2 \quad$ Switching between the constrained and unconstrained laws $\ldots \ldots \ldots$

5.3 Numerical Simulations $\ldots \ldots \ldots$. . . . . . . . . . . . . . . . . 40

5.3 .1 Monte Carlo simulation . . . . . . . . . . . . . . . . 40

$5.3 .2 \quad$ Tracking Problem With Constrained-Unconstrained Switching . . . . . . . . 43

\begin{tabular}{llr}
\hline 6 & Conclusions & 46
\end{tabular}

$\begin{array}{ll}\text { Bibliography } & 48\end{array}$

\section{Appendix}

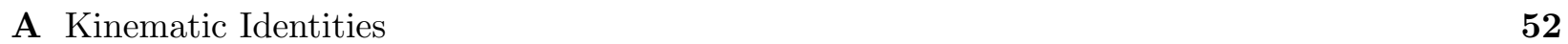

A.1 Transport Theorem . . . . . . . . . . . . . . . . . . . . . 52

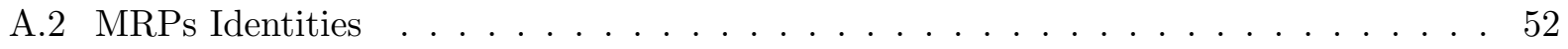

\begin{tabular}{|lc}
\hline B Lyapunov Theory & 54
\end{tabular}

B.1 Lyapunov Stability . . . . . . . . . . . . . . . . . . . . . 54

B.2 Lyapunov's Direct Method . . . . . . . . . . . . . . . . . . . . . 55

B.3 Constrained Lyapunov Function $\ldots \ldots \ldots \ldots$ 


\section{Tables}

\section{Table}

1.1 Comparison between existing constrained attitude control frameworks. $\ldots$. . . . . . 7

4.1 Simulation parameters. . . . . . . . . . . . . . . . . . . . 30

4.2 Reference frame parameters. . . . . . . . . . . . . . . . . 35 


\section{Figures}

\section{Figure}

1.1 Attitude exclusion constraint example. The boresight body-fixed vector of a camera $\hat{\boldsymbol{b}}$ is required to stay out of an exclusion cone around the inertially-fixed sun vector $\hat{\boldsymbol{n}}$. 2

1.2 The idea behind the geometric approach by Hablani $[8]$ is to compute an intermediate attitude to avoid the constraints. The figure shows an azimuth-elevation plane in which the unconstrained path is a simple slew azimuth maneuver (dash line). An intermediate attitude is computed to avoid the constraint. . . . . . . . . . . . . . . 4

$1.3 \quad$ SAMPEX avoids aligning its Heavy Ion Large Telescope with the velocity vector. . . 5

1.4 Some spacecraft using constrained attitude control. . . . . . . . . . . . . . . 6

2.1 Control system block diagram using a steering law. . . . . . . . . . . . . . 13

3.1 Conic constraint geometry $\ldots \ldots \ldots \ldots \ldots \ldots \ldots$

4.1 With perfect symmetry $\boldsymbol{v}_{R}$ can be $0 . \ldots \ldots \ldots \ldots \ldots$

4.2 Regulation control performance illustration: Typical slew maneuver. . . . . . . . . . 32

4.3 Regulation control performance illustration: Saddle-point avoidance. . . . . . . . . . 34

4.4 Tracking control performance illustration. . . . . . . . . . . . . . . 36

5.1 Worst-case scenario. The spacecraft is rotating in a fixed plane at maximum angular velocity straight into a constraint. $u_{\max }$ is the maximum torque capacity in that

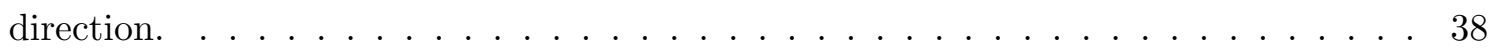


5.2 Monte Carlo simulation. . . . . . . . . . . . . . . . . . . . . . 42

5.3 Tracking control performance illustration using contrained-unconstrained switching. 44

5.4 Tracking control performance illustration using contrained-unconstrained switching. Exclusion constraint angles. Exclusion zones 3 and 4 are not considered by Algorithm $|2|$ because they are "far" from the trajectory. . . . . . . . . . . . . . . . . 45 


\section{Chapter 1}

\section{Introduction}

\section{$1.1 \quad$ Motivation}

The spacecraft's ability to align its orientation relative to a reference frame, or orientationunconstrained autonomous attitude control, has been extensively addressed in literature. In fact, the problem has been studied using several different frameworks. Linear and non-linear control[ 5 , 29, 34, 38, 49], optimal control [9, 12, sliding-mode control [26, 50], and adaptive control [35, 40] are only some of the techniques that have been successfully applied to the solution of this problem. It can be thus said that autonomous attitude control without orientation constraints is a mature technology.

However, spacecraft reorientation may have several design-specific pointing restrictions. These attitude constraints can be in the form of undesirable pointing regions. An example is any spacecraft carrying heat or light sensitive instruments, such as telescopes or cameras, that cannot be exposed to direct sunlight. Bright celestial objects may thus impose constraints to a slew maneuver. On the other hand, pointing restrictions can also manifest themselves as inclusive heading regions. For example, a change in attitude could be performed while keeping certain instruments, e.g. antennas or solar panels, pointing into a definite region in space. Ultimately, attitude constraints can be viewed as either exclusion or inclusion zones, usually defined by cones in space around either a forbidden or a mandatory nominal direction. An exclusion constraint example is depicted in Figure 1.1. The boresight body-fixed vector of a camera $\hat{b}$ is required to stay out of an exclusion cone around the inertially-fixed sun vector $\hat{\boldsymbol{n}}$. 


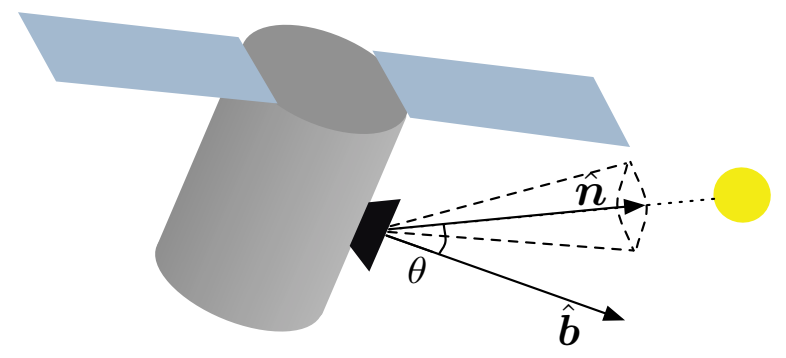

Figure 1.1: Attitude exclusion constraint example. The boresight body-fixed vector of a camera $\hat{\boldsymbol{b}}$ is required to stay out of an exclusion cone around the inertially-fixed sun vector $\hat{\boldsymbol{n}}$.

In spite of the fact that most mission requirements include some kind of constraints on slew maneuvers, the autonomous constrained attitude control literature is still sparse and many spacecraft perform constraint avoidance non-autonomously, through open-loop attitude way point navigation solutions, or semi-autonomously, using simple constraint detection algorithms. Autonomous constrained spacecraft attitude control is, therefore, not a mature technology.

This thesis proposes a novel autonomous attitude control algorithm that enforces an arbitrary number of inclusion and exclusion conic constraints. The technique is suitable for both the regulation (constant reference) and the tracking problem (continuously moving reference). Lyapunov theory is utilized to synthesize a mathematically tractable kinematic steering control law that allows attitude control with bounded angular velocity. By describing the orientation of a spacecraft using Modified Rodrigues Parameters (MRPs), the short slew path can always be followed. The problem of control torque saturation is addressed by investigating solutions to guarantee the constraint compliance even if the control torque actuation is limited.

Kinematic, or attitude-based, steering laws permit dividing the attitude and angular velocity control strategies into two completely separate loops, simplifying the synthesis of control laws 37 , 36. Using this scheme, an angular velocity loop, usually known as servo sub-system, is controlled by a kinematic loop. The benefit of such attitude steering laws is that the angular velocity vector is treated as the control vector, and the rate response due to a tracking can be shaped in very general ways. In particular, the implementation discussed in Reference [37] uses a smoothly saturated rate 
behavior. Thus, even with very large attitude tracking errors, the spacecraft closed loop response reaches a predicable upper rate limit.

The Modified Rodrigues Parameters (MRPs) constitute a singular, non-unique and minimal attitude representation[27]. The non-uniqueness can be used to switch the parameters at the unit sphere in order to avoid their only singularity while naturally overcoming the unwinding phenomenon[2], making a control scheme to always follow the shortest path[36]. These properties make the MRPs an elegant attitude representation.

Non-linear control methods using Lyapunov theory[13] have the advantage that they allow synthesizing relatively simple control laws, i.e. control laws given by closed-form, usually analytic, functions. This fact makes these algorithms specially suitable for on-board implementation. Lyapunov theory has been applied before to the constrained attitude control regulation problem using Euler angles[28] and quaternions[23, 25, 52]. However, it has not yet been applied to solve the attitude tracking problem with constraints. Furthermore, the novel use of a kinematic steering law allows the utilization of rate saturating functions to control the maximum angular velocity. Additionally, no previous work has analyzed how constraint avoidance and reaction wheel torque saturation are related. Finally, the use of MRPs in this problem formulation is also novel in that the solution provides a minimal attitude parameterization that yields globally non-singular behavior.

\subsection{Existing Constrained Attitude Control Frameworks}

The existing techniques for studying the constrained attitude control problem may be classified into six different groups. For the purpose of this section, a constraint is classified as kinematic hard, if it only depends on the current attitude; kinematic soft, if it depends on the attitude history; or dynamic, if it depends on the attitude and a moving reference[17. A conic constraint is a particular kinematic hard constraint defined by a security cone about a forbidden direction. Detailed constraint description is given in Chapter 3 .

The first framework relies on the geometry of the problem. In Reference [8], geometric relations are used to determine trajectories that avoid the constraint volumes [8, 44. In order to 


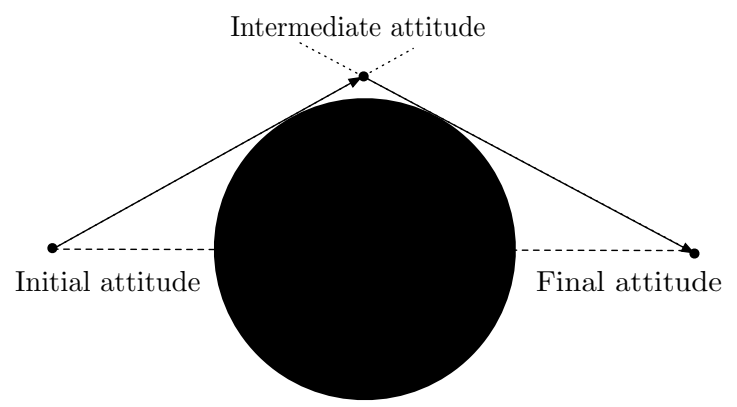

Figure 1.2: The idea behind the geometric approach by Hablani $[8$ is to compute an intermediate attitude to avoid the constraints. The figure shows an azimuth-elevation plane in which the unconstrained path is a simple slew azimuth maneuver (dash line). An intermediate attitude is computed to avoid the constraint.

solve the slew problem of rotating a spacecraft from an initial to a final orientation, these algorithms pre-compute a feasible trajectory through an intermediate attitude such that the exclusion constraints are not violated. A simple example is sketched in Figure 1.2. These techniques are relatively simple and can be used to slew a spacecraft from an initial to a final orientation under inclusion and exclusion constraints, but do not scale well when the number of constraints grow [17].

A different geometric approach has been used in the SAMPEX (Solar, Anomalous, and Magnetospheric Particle EXplorer) mission (see Figures 1.3 and 1.4a) in order to avoid its Heavy Ion Large Telescope (HILT) to align, within a certain angle, with the velocity vector, a condition that would have maximized the flux of orbital debris and micrometeroids into the instrument, reducing its lifetime[6]. SAMPEX used a very simple constraint avoidance technique. The guidance system checked the angle between the pointing target and the velocity vector. If it determined that the boresight vector of the HILT had entered the conic constraint, it would redefine its target outside the cone[6]. This approach is reactive and convergence cannot be demonstrated.

Another different geometric approach has been recently developed[]. The attitude slew problem is modeled as an optimal boundary value problem. Small forbidden regions can be avoided by an iterative process in which, by changing some constants, the resulting trajectory is evaluated in order to detect if the exclusion zones are effectively avoided. This technique can solve the exclusion constrained regulation problem. However, it has some drawbacks: it is not defined any measure to 


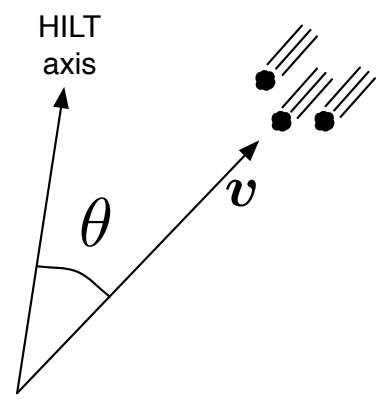

Figure 1.3: SAMPEX avoids aligning its Heavy Ion Large Telescope with the velocity vector.

evaluate how "small" a constraint really is and the iterating (not always convergent) process does not seem to make the algorithm suitable for on-board implementation.

The Constraint Monitor Algorithm (CMT) is a technique that uses a predictor-corrector approach to change the trajectory in real time when approaching a constraint[17]. As the name implies, CMT is always monitoring the control command of the attitude controller and extrapolating the current attitude forward. In case of a foreseen exclusion constraint violation, the spacecraft is rotationally accelerated in the radially opposite direction [43. The method, created for the Cassini mission [1, 43, has also been used in Deep-Space 1[16] (Figures 1.4b and 1.4c). CMT can be utilized to avoid all kinds of exclusion zones and, even though it was designed for performing a slew maneuver, it could be extended to work with a moving attitude reference. Unfortunately, the convergence of the algorithm cannot be demonstrated in the general case [17.

Randomized motion planning algorithms use graphs and random search to go from an initial to a final attitude avoiding all constraints[7]. The approach is as follows: from a vertex of the graph (state), a random search is performed to determine a set of feasible vertices, picking the one that minimizes some cost function. The technique solves the regulation problem with both exclusion and inclusion constraints[17]. However, it has mainly two drawbacks: convergence can be guaranteed only in a probabilistic sense and computational time grows dramatically with the size of the graph.

Optimization techniques can be applied to solve the problem of slewing a spacecraft from 


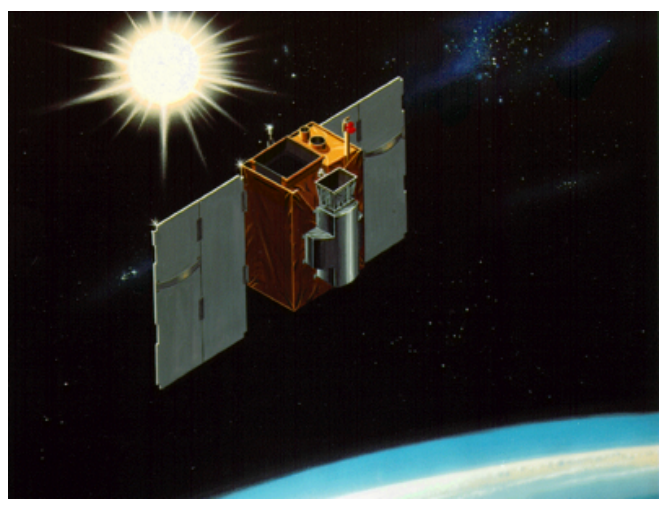

(a) SAMPEX (Geometric approach).

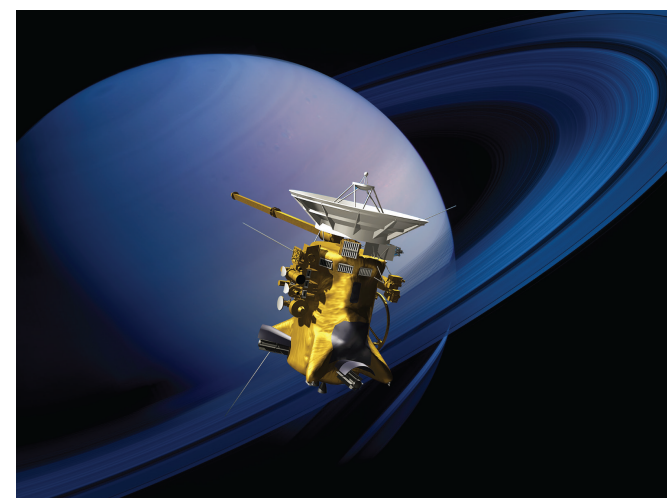

(b) Cassini (CMT).

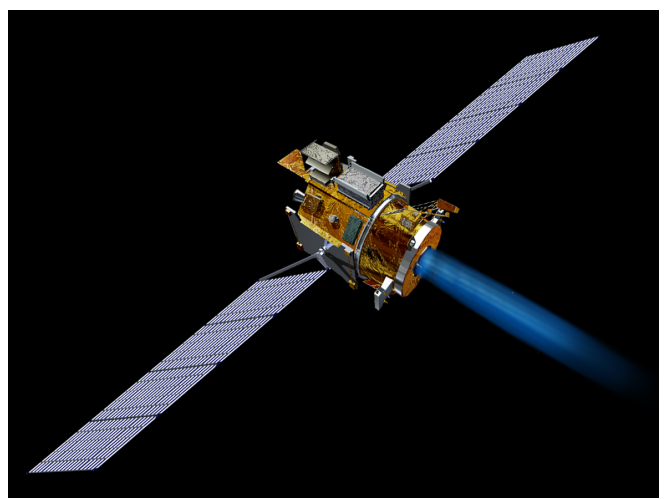

(c) Deep-Space 1 (CMT).

Figure 1.4: Some spacecraft using constrained attitude control.

an initial to a final state under constraints with bounded control torque and bounded angular rate, minimizing some functional, such as control effort or power. This is a hard-to-solve NonLinear optimization Problem (NLP) [24]. However, the formulation can be transformed into a nonconvex Quadratically Constrained Quadratic Programming (QCQP) problem[4] using quaternions, and further simplified using a semidefinite relaxation[45]. The problem can also be convexified as a Semi-Definite Programming (SDP) optimization problem using a different approach[15, 17]. Convex SDP problems are easier to solve than NLP[4]. The advantage of these methods is that they can solve problems with topologically different constraints. However, they are not specially suited for the tracking problem, with a continuously moving reference trajectory. Furthermore, since the approach is open-loop, constraint enforcement cannot be guaranteed under perturbations. 
Table 1.1: Comparison between existing constrained attitude control frameworks.

\begin{tabular}{c|c|c|c|c|c|c}
\hline \hline Method & Constraints & Complexity & $\begin{array}{c}\text { Bounded } \\
\text { Velocity }\end{array}$ & $\begin{array}{c}\text { Bounded } \\
\text { Torque }\end{array}$ & Tracking & Convergence \\
\hline \hline Geometric & Conic & Medium & Yes & Yes & No & Yes \\
\hline CMT & Arbitrary & Low & Yes & Yes & $\begin{array}{c}\text { May be } \\
\text { extended }\end{array}$ & Not shown \\
\hline $\begin{array}{c}\text { Random } \\
\text { Search }\end{array}$ & Arbitrary & High & Yes & Yes & No & Probabilistic \\
\hline $\begin{array}{c}\text { Optimal } \\
\text { Control }\end{array}$ & Convex & High & Yes & Yes & $\begin{array}{c}\text { May be } \\
\text { extended }\end{array}$ & Yes \\
\hline $\begin{array}{c}\text { Graph } \\
\text { Search }\end{array}$ & $\begin{array}{c}\text { Static } \\
\text { hard }\end{array}$ & High & Yes & Yes & No & Yes \\
\hline Lyapunov & Conic & Low & No & No & No & Yes \\
\hline \hline
\end{tabular}

Fortunately, Receding Horizon Control (RHC) [20] can be utilized to synthesize a closed-loop control law 11. Moreover, RHC might be used to solve the tracking problem. However, the technique is extremely expensive in terms of computational resources 30$]$.

A recently developed new framework divides the attitude space into discrete cells and uses graph searching algorithms, like $A^{*}$, to find an optimal solution to the constrained problem[18, 46. One of the advantages of this technique is that the algorithm becomes more efficient as more exclusion constraints are added[18]. The method, however, is not suited for the tracking problem.

Potential function-based algorithms use Lyapunov theory to design control laws that converge to the target while evading constraints. This approach has been proposed with singular Euler angles 28] and the unit-constrained quaternions [23, 25] to solve the constrained regulation problem and, in the last case, with any number of exclusion and inclusion constraints. In Reference [52], another method using quaternions and a Hamiltonian formulation is proposed to handle one exclusion constraint using Lyapunov functions based on geometric considerations. However, it cannot be shown to converge, and may not even be feasible, with several exclusion constraints.

The main advantage of using Lyapunov theory is that the synthesized control laws are mathematically tractable. However, the method cannot handle arbitrarily-shaped constraints. Furthermore, none of the existing Lyapunov-derived algorithms is torque-bounded. 
Table 1.1 shows a general comparison of the existing frameworks. The Complexity column loosely describes the algorithmic numerical complexity. A low complexity means that only a few function evaluations are needed. A high complexity, on the other side, means that more complex numerical algorithms are required, like optimization algorithms or graph search, with lots of function evaluations. The optimization approach can manage several types of constraints, as long as they can be convexified [4].

\subsection{Thesis Research Goals}

Table 1.1 gives a good comparison between some of the pros and cons of each framework. The optimal control approach and graph search methods seem to be the best suited to solve the constrained orientation control problem. However, the first is open-loop (non-robust) and neither of both solve the tracking problem. Moreover, both have high algorithmic complexity. On the other hand, the advantage of Lyapunov methods is simplicity in the synthesized closed-loop control laws. However, the existing techniques for constrained attitude control derived using Lyapunov theory are neither torque-bounded nor velocity-bounded. Furthermore, they do not solve the tracking problem either.

The main goal of this thesis is to develop a Lyapunov-derived control law such that

(1) Solves the slew problem from an initial to a final fixed orientation under any number of feasible exclusion and inclusion conic constraints with a bounded velocity and bounded control torque. The closed-loop solution must be robust against unmodeled torques.

(2) Solves the tracking control problem of following a continuously moving attitude trajectory under any number of feasible exclusion and inclusion conic constraints.

(3) Uses Modified Rodrigues Parameters (MRPs) as attitude descriptors. MRPs have not been used yet to parameterize the problem and have several advantages: minimal, non-singular, and describe short rotations. 
The thesis is organized as follows. In Chapter 2, the rate-limited Lyapunov-derived unconstrained steering law is shown. A brief presentation of MRPs and the kinetics of a spacecraft with reaction wheels, used to mathematically synthesized the law, is given. In Chapter 3, a discussion and classification of orientation constraints are given, showing a novel description of conic constraints as functions of MRPs. The regulation and tracking problem with inclusion and exclusion conic constraints are developed in Chapter 4. Chapter 5 shows the effect of a bounded control torque, giving an algorithm for switching between the unconstrained and constrained laws when far from exclusion constraints. Finally, Chapter 6 discusses the conclusions and possibilities for future work. 


\section{Chapter 2}

\section{Unconstrained Steering Law Using Modified Rodrigues Parameters}

The purpose of this Chapter is threefold. The main goal is to introduce a kinematic steering law that allows regulation and tracking of a spacecraft using two different nested control laws: an inner servo-subsystem that controls angular velocity and an outer loop, attitude-based, that steers attitude using angular velocity as a control variable. Since the control law utilizes Modified Rodrigues Parameters (MRPs) as kinematic states and Reaction Wheels (RW) as actuators, the spacecraft's kinematics using MRPs and kinetics using RW are briefly reviewed.

Constraints are not considered in this Chapter. The control law described is orientation unconstrained. However, it is the basis to the constrained-orientation control algorithms discussed in Chapter 4

\subsection{Modified Rodrigues Parameters}

The MRPs are a minimal parametrization set of the rotation group $\mathrm{SO}(3)$. The MRP vector

$\boldsymbol{\sigma}$ is defined in terms of the quaternion $\boldsymbol{\beta}=\left[\begin{array}{llll}\beta_{0} & \beta_{1} & \beta_{2} & \beta_{3}\end{array}\right]^{T}$ or the principal rotation vector representation $(\hat{\boldsymbol{e}}, \Phi)$ as [51, 27, 42, 36]

$$
\boldsymbol{\sigma}=\frac{1}{1+\beta_{0}}\left[\begin{array}{lll}
\beta_{1} & \beta_{2} & \beta_{3}
\end{array}\right]^{T}=\tan \left(\frac{\Phi}{4}\right) \hat{\boldsymbol{e}}
$$

where $\beta_{0}$ represents the scalar part of the quaternion, $\hat{\boldsymbol{e}}$ is the principal rotation axis, and $\Phi$ is the principal rotation angle. The representation is singular whenever $\beta_{0}=-1$, where the rotation angle is $\Phi= \pm 360^{\circ}$. 
MRPs have a very interesting property as the result of a stereographic projection parameters of the four-dimensional quaternion unit sphere onto a three-dimensional hyperplane [27, 48].

The MRPs, as the quaternions, are not unique. In fact, since $\boldsymbol{\beta}$ and $-\boldsymbol{\beta}$ represent the same attitude, $\boldsymbol{\sigma}$ and $\boldsymbol{\sigma}^{s}$, known as the shadow set, also represent the same orientation, where [36, 10]

$$
\boldsymbol{\sigma}^{s}=-\frac{1}{1-\beta_{0}}\left[\begin{array}{lll}
\beta_{1} & \beta_{2} & \beta_{3}
\end{array}\right]^{T}=-\frac{\boldsymbol{\sigma}}{\boldsymbol{\sigma}^{T} \boldsymbol{\sigma}}
$$

Equation (2.1) shows that short rotations $\left(\Phi \leq 180^{\circ}\right)$ have $|\boldsymbol{\sigma}| \leq 1$. Using this fact and the shadow set given in Equation (2.2), the general approach is to switch between MRP representations at the unit sphere in order to avoid the singularity while always describing short rotations [36].

The rotation matrix $[C(\boldsymbol{\sigma})]$, also represented as $[P Q]$ when describes the orientation of a frame $\mathcal{P}$ relative to a frame $\mathcal{Q}$, can be computed from the $\operatorname{MRP} \boldsymbol{\sigma}\left(\right.$ or $\boldsymbol{\sigma}_{\mathcal{P} / \mathcal{Q}}$ ) as 36

$$
[C(\boldsymbol{\sigma})]=\left[I_{3 \times 3}\right]+\frac{8[\tilde{\boldsymbol{\sigma}}]^{2}-4\left(1-\boldsymbol{\sigma}^{T} \boldsymbol{\sigma}\right)[\tilde{\boldsymbol{\sigma}}]}{\left(1+\boldsymbol{\sigma}^{T} \boldsymbol{\sigma}\right)^{2}}
$$

where $[\tilde{\boldsymbol{\sigma}}]$ is the associated skew-symmetric matrix 36$]$

$$
\tilde{\boldsymbol{\sigma}}=\left[\begin{array}{ccc}
0 & -\sigma_{3} & \sigma_{2} \\
\sigma_{3} & 0 & -\sigma_{1} \\
-\sigma_{2} & \sigma_{1} & 0
\end{array}\right]
$$

Even though there exists a direct transformation from Direction Cosine Matrix (DCM) into MRPs, the mapping is singular for rotations of $180^{\circ}$ [36]. Therefore, Sheppard's method can be used to compute quaternions from DCM[41] and then Equation (2.1) can be utilized to calculate the MRPs.

The MRPs kinematic differential equation is given by 36.

$$
\dot{\boldsymbol{\sigma}}=\frac{1}{4}\left[\left(1-\boldsymbol{\sigma}^{T} \boldsymbol{\sigma}\right)\left[I_{3 \times 3}\right]+2[\tilde{\boldsymbol{\sigma}}]+2 \boldsymbol{\sigma} \boldsymbol{\sigma}^{T}\right] \boldsymbol{\omega}=\frac{1}{4}[B(\boldsymbol{\sigma})] \boldsymbol{\omega}
$$

If $\boldsymbol{\sigma}$ represents the attitude of frame $\mathcal{P}$ relative to $\mathcal{Q}$ (noted as $\boldsymbol{\sigma}_{\mathcal{P} / \mathcal{Q}}$ ), then $\boldsymbol{\omega}$ is the angular velocity of frame $\mathcal{P}$ relative to $\mathcal{Q}$ written in $\mathcal{P}$-frame components (also noted as ${ }^{\mathcal{P}} \boldsymbol{\omega}_{\mathcal{P} / \mathcal{Q}}$, where the left superscript notes the frame with respect to which the vector components are taken). 


\section{$2.2 \quad$ Rigid-Body Dynamics with Reaction Wheels}

The rotational equations of motion of a rigid spacecraft with $N_{\mathrm{RW}}$ perfectly symmetric and balanced Reaction Wheels (RW) are given by 36$]$

$$
\left[I_{\mathrm{RW}}\right] \dot{\boldsymbol{\omega}}=-[\tilde{\boldsymbol{\omega}}]\left(\left[I_{\mathrm{RW}}\right] \boldsymbol{\omega}+\left[G_{\mathrm{S}}\right] \boldsymbol{h}_{\mathrm{s}}\right)-\left[G_{\mathrm{s}}\right] \boldsymbol{u}_{\mathrm{s}}+\boldsymbol{L}
$$

where $\left[I_{\mathrm{RW}}\right]$ is

$$
\left[I_{\mathrm{RW}}\right]=\left[I_{\mathrm{S}}\right]+\sum_{i=1}^{N_{\mathrm{RW}}}\left(J_{t_{i}} \hat{\boldsymbol{g}}_{t_{i}} \hat{\boldsymbol{g}}_{t_{i}}^{T}+J_{g_{i}} \hat{\boldsymbol{g}}_{g_{i}} \hat{\boldsymbol{g}}_{g_{i}}^{T}\right)
$$

and the projection matrix $\left[G_{\mathrm{S}}\right]$ is

$$
\left[G_{\mathrm{s}}\right]=\left[\begin{array}{lllll}
\hat{\boldsymbol{g}}_{s_{1}} & \ldots & \hat{\boldsymbol{g}}_{s_{i}} & \ldots & \hat{\boldsymbol{g}}_{s_{N_{\mathrm{RW}}}}
\end{array}\right]
$$

The RW angular momentum vector $\boldsymbol{h}_{\mathrm{s}}$ is computed as

$$
\boldsymbol{h}_{\mathrm{s}}=\left[\begin{array}{lllll}
J_{s_{1}}\left(\hat{\boldsymbol{g}}_{s_{1}}^{T} \boldsymbol{\omega}+\Omega_{1}\right) & \ldots & J_{s_{i}}\left(\hat{\boldsymbol{g}}_{s_{i}}^{T} \boldsymbol{\omega}+\Omega_{i}\right) & \ldots & J_{s_{N}}\left(\hat{\boldsymbol{g}}_{s_{N_{\mathrm{RW}}}^{T}}^{T} \boldsymbol{\omega}+\Omega_{N_{\mathrm{RW}}}\right)
\end{array}\right]^{T}
$$

$\left[I_{\mathrm{S}}\right]$ is the inertia tensor of the system with the wheels considered as point masses. A principalaxis frame $\mathcal{W}_{i}:\left\{\hat{\boldsymbol{g}}_{s_{i}}, \hat{\boldsymbol{g}}_{t_{i}}, \hat{\boldsymbol{g}}_{g_{i}}\right\}$ is attached to each RW, where $\hat{\boldsymbol{g}}_{s_{i}}$ is the direction of the spin axis. $\left[I_{w_{i}}\right]=\operatorname{diag}\left(J_{s_{i}}, J_{t_{i}}, J_{g_{i}}\right)$ is the inertia matrix of each wheel written in the $\mathcal{W}$ frame relative to its center of mass. $\Omega_{i}$ is the angular velocity of the RW $i$ relative to the spacecraft. The vector $\boldsymbol{u}_{\mathrm{s}}$ contains the torques applied to each RW axis. The RW torque may saturate, with a maximum torque $u_{s_{\max }} . \boldsymbol{L}$ is the resultant external perturbation torque applied to the spacecraft.

The vector $\boldsymbol{\omega}$ is a shorthand notation for $\boldsymbol{\omega}_{\mathcal{B} / \mathcal{N}}$, where $\mathcal{B}$ is a body-fixed frame and $\mathcal{N}$ is an inertial frame. The over-dot symbol $(\bullet)$ represents an inertial derivative while the prime symbol $(\bullet ')$ represents a derivative relative to the rotating body frame. Although the equations of motion can be solved in any frame, it is assumed that every vector and tensor are written in the body-fixed frame $\mathcal{B}$.

These are the dynamic equations of motion that are used in the rest of this work. 


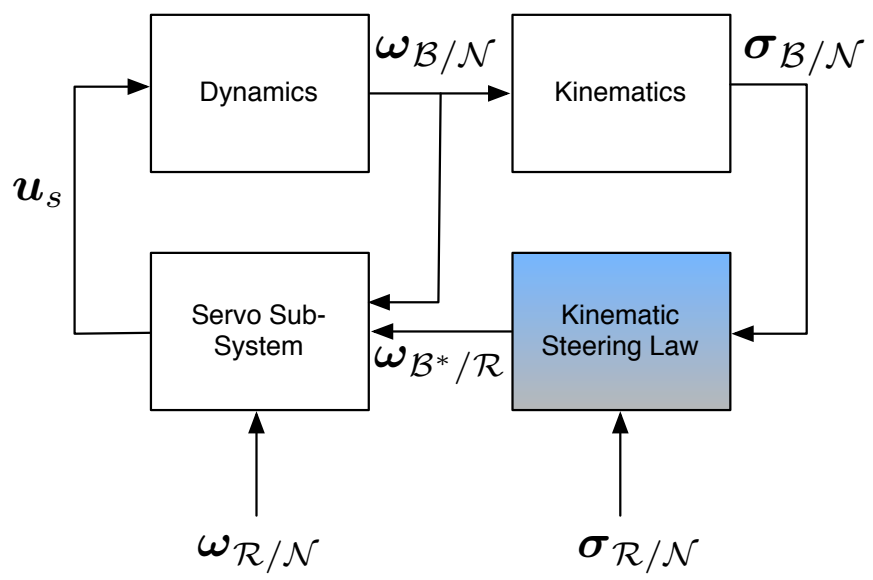

Figure 2.1: Control system block diagram using a steering law.

\subsection{Unconstrained MRP Steering Law}

The unconstrained attitude problem can be solved using the Lyapunov direct method and two separate feedback loops for attitude and rate tracking[37], in a rather similar way as the Backstepping Control method[13, 14] provides a cascaded control design. This approach can be used with cascaded systems where the output of one sub-system is the input of the next. Spacecraft's kinetics and kinematics form one of such particular systems.

A high-level block diagram is shown in Figure 2.1. The block Dynamics is the spacecraft with RW dynamics given in Equation (2.6). The block Kinematics represents the kinematic differential equation given in Equation (2.5). An inner Servo Sub-System loop controls angular velocity, having as inputs the angular velocity of the reference frame $\mathcal{R}$ relative to inertial frame $\mathcal{N}, \boldsymbol{\omega}_{\mathcal{R} / \mathcal{N}}$, and the ouput of the steering law, the desired body frame $\mathcal{B}^{*}$ rate relative to the reference, $\boldsymbol{\omega}_{\mathcal{B}^{*} / \mathcal{R}}$. The output of the servo is the torque vector control of the RWs, $\boldsymbol{u}_{\mathrm{s}}$. An outer, narrower-bandwith loop, or Kinematic Steering Law, controls attitude, taking the body frame $\mathcal{B}$ attitude relative to inertial $\mathcal{N}, \boldsymbol{\sigma}_{\mathcal{B} / \mathcal{N}}$, and the reference frame attitude relative to inertial, $\boldsymbol{\sigma}_{\mathcal{R} / \mathcal{N}}$, as inputs.

Using two different loops for attitude and angular velocity has the following advantages. First, the kinematic model given by Equation (2.5) is exact. Second, the synthesis of control laws is simplified. Third, as this thesis demonstrates, the angular velocity servo loop does not need to 
be changed when conic constraints are added.

The goal of the control scheme is to steer the body frame $\mathcal{B}$ to the reference frame $\mathcal{R}$. In other words, the relative attitude $\boldsymbol{\sigma}_{\mathcal{B} / \mathcal{R}}$ and angular velocity $\boldsymbol{\omega}_{\mathcal{B} / \mathcal{R}}$ are to be driven to zero.

\subsubsection{MRP Steering Law}

Consider the following Lyapunov candidate function 36 ]

$$
V\left(\boldsymbol{\sigma}_{\mathcal{B} / \mathcal{R}}\right)=2 \ln \left(1+\boldsymbol{\sigma}_{\mathcal{B} / \mathcal{R}}^{T} \boldsymbol{\sigma}_{\mathcal{B} / \mathcal{R}}\right)
$$

Taking time derivatives

$$
\dot{V}\left(\boldsymbol{\sigma}_{\mathcal{B} / \mathcal{R}}\right)=\frac{4 \boldsymbol{\sigma}_{\mathcal{B} / \mathcal{R}}^{T} \dot{\boldsymbol{\sigma}}_{\mathcal{B} / \mathcal{R}}}{1+\boldsymbol{\sigma}_{\mathcal{B} / \mathcal{R}}^{T} \boldsymbol{\sigma}_{\mathcal{B} / \mathcal{R}}}
$$

Using Equation (2.5) and the fact ${ }^{1}$ that $\boldsymbol{\sigma}_{\mathcal{B} / \mathcal{R}}^{T}\left[\tilde{\boldsymbol{\sigma}}_{\mathcal{B} / \mathcal{R}}\right]=0$, it can be immediately shown that

$$
\dot{V}\left(\boldsymbol{\sigma}_{\mathcal{B} / \mathcal{R}}\right)=\boldsymbol{\sigma}_{\mathcal{B} / \mathcal{R}}^{T} \boldsymbol{\omega}_{\mathcal{B} / \mathcal{R}}
$$

Let $\mathcal{B}^{*}$ be the desired body orientation and $\boldsymbol{\omega}_{\mathcal{B}^{*} / \mathcal{R}}$ the desired angular velocity vector at which the spacecraft body should be rotating relative to the reference orientation. In the following steering law $\boldsymbol{\omega}_{\mathcal{B}^{*} / \mathcal{R}}$ is treated as a control variable, assuming an inner rate servo loop exists that implements these speeds. The kinematic steering command is expressed as

$$
\omega_{\mathcal{B}^{*} / \mathcal{R}}=-\boldsymbol{f}\left(\boldsymbol{\sigma}_{\mathcal{B} / \mathcal{R}}\right)
$$

where $\boldsymbol{f}\left(\boldsymbol{\sigma}_{\mathcal{B} / \mathcal{R}}\right)$ is an odd function such that

$$
\boldsymbol{\sigma}_{\mathcal{B} / \mathcal{R}}^{T} \boldsymbol{f}\left(\boldsymbol{\sigma}_{\mathcal{B} / \mathcal{R}}\right)>0
$$

The Lyapunov function rate will, thus, be negative definite, and the system, globally asymptotically stable 2

$$
\dot{V}\left(\boldsymbol{\sigma}_{\mathcal{B} / \mathcal{R}}\right)=-\boldsymbol{\sigma}_{\mathcal{B} / \mathcal{R}}^{T} \boldsymbol{f}\left(\boldsymbol{\sigma}_{\mathcal{B} / \mathcal{R}}\right)<0 \quad \forall \boldsymbol{\sigma}_{\mathcal{B} / \mathcal{R}} \neq \mathbf{0}
$$

\footnotetext{
${ }^{1}$ See Appendix A for details.

${ }^{2}$ See Appendix $\bar{B}$ for a brief introduction to Lyapunov theory.
} 
In this work, the smoothly saturated odd function given by

$$
f\left(x_{i}\right)=\frac{2 \omega_{\max }}{\pi} \arctan \left(\frac{\pi}{2 \omega_{\max }}\left(K_{1} x_{i}+K_{3} x_{i}^{3}\right)\right) \quad i=1,2,3
$$

is used, where $\boldsymbol{f}\left(\boldsymbol{\sigma}_{\mathcal{B} / \mathcal{R}}\right)=\left[\begin{array}{lll}f\left(\sigma_{1}\right) & f\left(\sigma_{2}\right) & f\left(\sigma_{3}\right)\end{array}\right]^{T}$.

\subsubsection{Servo Sub-System}

A servo sub-system is utilized to produce the required torques to make the body rates track the desired body rates commanded by the steering law. The tracking error is defined as

$$
\omega_{\mathcal{B} / \mathcal{B}^{*}}=\omega_{\mathcal{B} / \mathcal{N}}-\omega_{\mathcal{B}^{*} / \mathcal{N}}=\omega_{\mathcal{B} / \mathcal{N}}-\omega_{\mathcal{B}^{*} / \mathcal{R}}-\omega_{\mathcal{R} / \mathcal{N}}
$$

where the fact that $\boldsymbol{\omega}_{\mathcal{B}^{*} / \mathcal{N}}=\boldsymbol{\omega}_{\mathcal{B}^{*} / \mathcal{R}}+\boldsymbol{\omega}_{\mathcal{R} / \mathcal{N}}$ has been used. $\boldsymbol{\omega}_{\mathcal{B}^{*} / \mathcal{R}}$ is the kinematic steering rate command and $\boldsymbol{\omega}_{\mathcal{R} / \mathcal{N}}$ is an input coming from the attitude navigation solution. To create a rate-servo that is robust to unmodeled torques [36], the integral term $\boldsymbol{z}$ is defined as

$$
\boldsymbol{z}=\int_{t_{0}}^{t} \boldsymbol{\omega}_{\mathcal{B} / \mathcal{B}^{*}} \mathrm{~d} \tau
$$

Consider the Lyapunov candidate function 37.

$$
V_{\boldsymbol{\omega}}\left(\boldsymbol{\omega}_{\mathcal{B} / \mathcal{B}^{*}}, \boldsymbol{z}\right)=\frac{1}{2} \boldsymbol{\omega}_{\mathcal{B} / \mathcal{B}^{*}}^{T}\left[I_{\mathrm{RW}}\right] \boldsymbol{\omega}_{\mathcal{B} / \mathcal{B}^{*}}+\frac{1}{2} \boldsymbol{z}^{T}\left[K_{\mathrm{I}}\right] \boldsymbol{z}
$$

where $\left[K_{\mathrm{I}}\right]$ is a positive definite matrix. Thus

$$
\dot{V}_{\boldsymbol{\omega}}\left(\boldsymbol{\omega}_{\mathcal{B} / \mathcal{B}^{*}}, \boldsymbol{z}\right)=\boldsymbol{\omega}_{\mathcal{B} / \mathcal{B}^{*}}^{T}\left(\left[I_{\mathrm{RW}}\right] \boldsymbol{\omega}_{\mathcal{B} / \mathcal{B}^{*}}^{\prime}+\left[K_{\mathrm{I}}\right] \boldsymbol{z}\right)
$$

For convenience, body frame derivatives are taken in Equation 2.20).

Using the identities ${ }^{3} \boldsymbol{\omega}_{\mathcal{B} / \mathcal{N}}^{\prime}=\dot{\boldsymbol{\omega}}_{\mathcal{B} / \mathcal{N}}$ and $\boldsymbol{\omega}_{\mathcal{R} / \mathcal{N}}^{\prime}=\dot{\boldsymbol{\omega}}_{\mathcal{R} / \mathcal{N}}-\boldsymbol{\omega}_{\mathcal{B} / \mathcal{N}} \times \boldsymbol{\omega}_{\mathcal{R} / \mathcal{N}}$, and Equation 2.17

$$
\omega_{\mathcal{B} / \mathcal{B}^{*}}^{\prime}=\dot{\omega}_{\mathcal{B} / \mathcal{N}}-\omega_{\mathcal{B}^{*} / \mathcal{R}}^{\prime}-\dot{\omega}_{\mathcal{R} / \mathcal{N}}+\omega_{\mathcal{B} / \mathcal{N}} \times \omega_{\mathcal{R} / \mathcal{N}}
$$

Plugging Equation 2.21) into Equation 2.20

$$
\dot{V}_{\boldsymbol{\omega}}\left(\boldsymbol{\omega}_{\mathcal{B} / \mathcal{B}^{*}}, \boldsymbol{z}\right)=\boldsymbol{\omega}_{\mathcal{B} / \mathcal{B}^{*}}^{T}\left(\left[I_{\mathrm{RW}}\right] \dot{\boldsymbol{\omega}}_{\mathcal{B} / \mathcal{N}}-\left[I_{\mathrm{RW}}\right]\left(\boldsymbol{\omega}_{\mathcal{B}^{*} / \mathcal{R}}^{\prime}+\dot{\boldsymbol{\omega}}_{\mathcal{R} / \mathcal{N}}-\boldsymbol{\omega}_{\mathcal{B} / \mathcal{N}} \times \boldsymbol{\omega}_{\mathcal{R} / \mathcal{N}}\right)+\left[K_{\mathrm{I}}\right] \boldsymbol{z}\right)
$$

\footnotetext{
${ }^{3}$ See Appendix A
} 
Using the equations of motion given in Equation (2.6)

$$
\begin{array}{r}
\dot{V}_{\boldsymbol{\omega}}\left(\boldsymbol{\omega}_{\mathcal{B} / \mathcal{B}^{*}}, \boldsymbol{z}\right)=\boldsymbol{\omega}_{\mathcal{B} / \mathcal{B}^{*}}^{T}\left[-\left[\tilde{\boldsymbol{\omega}}_{\mathcal{B} / \mathcal{N}}\right]\left(\left[I_{\mathrm{RW}}\right] \boldsymbol{\omega}_{\mathcal{B} / \mathcal{N}}+\left[G_{\mathrm{s}}\right] \boldsymbol{h}_{\mathrm{s}}\right)-\left[G_{\mathrm{S}}\right] \boldsymbol{u}_{\mathrm{s}}+\boldsymbol{L}+\left[K_{\mathrm{I}}\right] \boldsymbol{z}\right. \\
\left.-\left[I_{\mathrm{RW}}\right]\left(\boldsymbol{\omega}_{\mathcal{B}^{*} / \mathcal{R}}^{\prime}+\dot{\boldsymbol{\omega}}_{\mathcal{R} / \mathcal{N}}-\boldsymbol{\omega}_{\mathcal{B} / \mathcal{N}} \times \boldsymbol{\omega}_{\mathcal{R} / \mathcal{N}}\right)\right]
\end{array}
$$

Forcing $\dot{V}_{\boldsymbol{\omega}}=-\boldsymbol{\omega}_{\mathcal{B} / \mathcal{B}^{*}}^{T}[P] \boldsymbol{\omega}_{\mathcal{B} / \mathcal{B}^{*}}$, with $[P]$ being a positive definite matrix, the kinetic system will be globally asymptotically stable. Equating

$$
\begin{aligned}
-[P] \boldsymbol{\omega}_{\mathcal{B} / \mathcal{B}^{*}}=\left[-\left[\tilde{\boldsymbol{\omega}}_{\mathcal{B} / \mathcal{N}}\right]\left(\left[I_{\mathrm{RW}}\right] \boldsymbol{\omega}_{\mathcal{B} / \mathcal{N}}+\left[G_{\mathrm{s}}\right] \boldsymbol{h}_{\mathrm{s}}\right)\right. & -\left[G_{\mathrm{s}}\right] \boldsymbol{u}_{\mathrm{s}}+\boldsymbol{L}+\left[K_{\mathrm{I}}\right] \boldsymbol{z} \\
& \left.-\left[I_{\mathrm{RW}}\right]\left(\boldsymbol{\omega}_{\mathcal{B}^{*} / \mathcal{R}}^{\prime}+\dot{\boldsymbol{\omega}}_{\mathcal{R} / \mathcal{N}}-\boldsymbol{\omega}_{\mathcal{B} / \mathcal{N}} \times \boldsymbol{\omega}_{\mathcal{R} / \mathcal{N}}\right)\right]
\end{aligned}
$$

Equivalently

$$
\left[G_{\mathrm{s}}\right] \boldsymbol{u}_{\mathrm{S}}=\boldsymbol{L}_{r}
$$

where

$$
\begin{aligned}
\boldsymbol{L}_{r}=[P] \boldsymbol{\omega}_{\mathcal{B} / \mathcal{B}^{*}}+\left[K_{\mathrm{I}}\right] \boldsymbol{z}-\left[\tilde{\boldsymbol{\omega}}_{\mathcal{B} / \mathcal{N}}\right]\left(\left[I_{\mathrm{RW}}\right] \boldsymbol{\omega}_{\mathcal{B} / \mathcal{N}}\right. & \left.+\left[G_{\mathrm{s}}\right] \boldsymbol{h}_{\mathrm{s}}\right) \\
& -\left[I_{\mathrm{RW}}\right]\left(\boldsymbol{\omega}_{\mathcal{B}^{*} / \mathcal{R}}^{\prime}+\dot{\boldsymbol{\omega}}_{\mathcal{R} / \mathcal{N}}-\boldsymbol{\omega}_{\mathcal{B} / \mathcal{N}} \times \boldsymbol{\omega}_{\mathcal{R} / \mathcal{N}}\right)
\end{aligned}
$$

Even though the RW torques can be mapped from $\boldsymbol{L}_{r}$ in several different ways, in this thesis the minimum norm inverse is used[36]

$$
\boldsymbol{u}_{\mathrm{s}}=\left[G_{\mathrm{s}}\right]^{T}\left(\left[G_{\mathrm{s}}\right]\left[G_{\mathrm{S}}\right]^{T}\right)^{-1} \boldsymbol{L}_{r}
$$

Equations (2.13) and (2.27) can be used to control a spacecraft's attitude using reaction wheels without constraints. As with any kinematic steering law, the rate-servo sub-system needs to have a faster frequency response to ensure overall stability.

\subsubsection{A Word on Numerical Computation}

The algorithm requires the numerical computation of the integral $\boldsymbol{z}=\int_{t_{0}}^{t} \boldsymbol{\omega}_{\mathcal{B} / \mathcal{B}^{*}} \mathrm{~d} \tau$ and the body frame derivative $\boldsymbol{\omega}_{\mathcal{B}^{*} / \mathcal{R}}^{\prime}$ 
The integral term is numerically computed using the very simple trapezoidal rule 33], which consists on interpolating the integrand with a linear function.

The numerical derivative, however, is much more delicate. In fact, depending on the frequency of the servo sub-system loop and the frequency content of $\boldsymbol{\omega}_{\mathcal{B}^{*} / \mathcal{R}}$, significant noise can be injected into the control loop, requiring some kind of filtering on the torque command signals of the RW. The approach taken here is to compute the numerical derivative using the noise-prone backward differences 33

$$
\boldsymbol{\omega}_{\mathcal{B}^{*} / \mathcal{R}}^{\prime}\left(t_{i}\right) \approx f_{\text {outer }}\left(\boldsymbol{\omega}_{\mathcal{B}^{*} / \mathcal{R}}\left(t_{i}\right)-\boldsymbol{\omega}_{\mathcal{B}^{*} / \mathcal{R}}\left(t_{i-1}\right)\right)
$$

where $f_{\text {outer }}$ is the frequency of the outer loop.

However, the derivative is smoothed using a simple moving average 32]. The width of the window can be adjusted as a function of $f_{\text {outer }}$.

Since $\boldsymbol{\omega}_{\mathcal{B}^{*} / \mathcal{R}}^{\prime}$ is a feed-forward term, its accuracy is not crucial. Indeed, it has been seen that, even in the case of over-filtering with a very wide window, the algorithm works well. 


\section{Chapter 3}

\section{Attitude Pointing Constraint Geometry Formulation}

This Chapter is divided into two parts. First, a brief overview of the type of orientation constraints that often appear in space missions is reviewed. In the second part, conic constraints are studied in detail, with particular emphasis on a formulation as a function of MRPs, since the rest of this work deals only with this specific type of orientation constraint.

\subsection{Constraint Classification}

Orientation constraints can be classified as inclusion or exclusion constraints. In the first group, a given body-fixed direction has to be maintained pointing inside a definite region in space while a maneuver is being performed. On the other hand, Kim, Meshabi, et. al. 17] classify exclusion constraints into four different types

(1) Type-I (static hard constraints): This is the most common type of constraint, in which "there is strict non-exposure constraints on the on-board sensitive instruments with respect to celestial objects" [17]. In other words, the current attitude cannot enter certain inertially fixed forbidden regions. In Reference [17], the categories conic and static hard constraints can be used interchangeably. However, it is possible to generalize Type-I constraints to any constraint that only depends on current attitude, being conic constraints a special case. Conic constraints are defined by a forbidden direction in space and a security cone around it given by a constant angle. The mathematical formulation of the restriction can be written 
as

$$
C\left(\boldsymbol{\sigma}_{\mathcal{B} / \mathcal{N}}\right)<0
$$

where $C\left(\sigma_{\mathcal{B} / \mathcal{N}}\right)$ is some function of the current attitude.

(2) Type-II (static soft constraints): This category is a relaxation of Type-I constraints in which violations of the forbidden zone are allowed, but for a limited amount of time. In other words, static soft constraints depend on attitude history. Mathematically

$$
\int_{t_{0}}^{t_{1}}\left|C\left(\boldsymbol{\sigma}_{\mathcal{B} / \mathcal{N}}\right)\right|<\phi
$$

where $\phi$ is a constant.

(3) Type-III (dynamic constraints): If the forbidden region changes with time, the constraint is dynamic. A dynamic conic constraint is one in which the axis is not inertially fixed. Dynamic constraints can also be hard or soft.

(4) Type-IV (mixed constraints): This category includes any possible combination of the last three.

\subsection{Conic Constraints}

Henceforth, only conic constraints are considered. This particular static soft constraint is illustrated in Figure 3.1, where an inertially fixed unit vector $\hat{\boldsymbol{n}}$ defines an exclusion or inclusion cone around it.

For an exclusion constraint, the goal is to slew a spacecraft avoiding a body-fixed unit vector $\hat{\boldsymbol{b}}$ entering the cone. The security angle is given by $\theta_{\text {min }}$, while $\theta$ is the instantaneous angle between both vectors. In a typical application $\hat{\boldsymbol{n}}$ can be a unit vector pointing towards the sun (approxi-

mately inertially fixed) while $\hat{\boldsymbol{b}}$ is the boresight vector of a camera (body-fixed). Mathematically, the condition is described as 1 ]

$$
\hat{\boldsymbol{n}} \cdot \hat{\boldsymbol{b}}=\cos (\theta)<\cos \left(\theta_{\min }\right)
$$



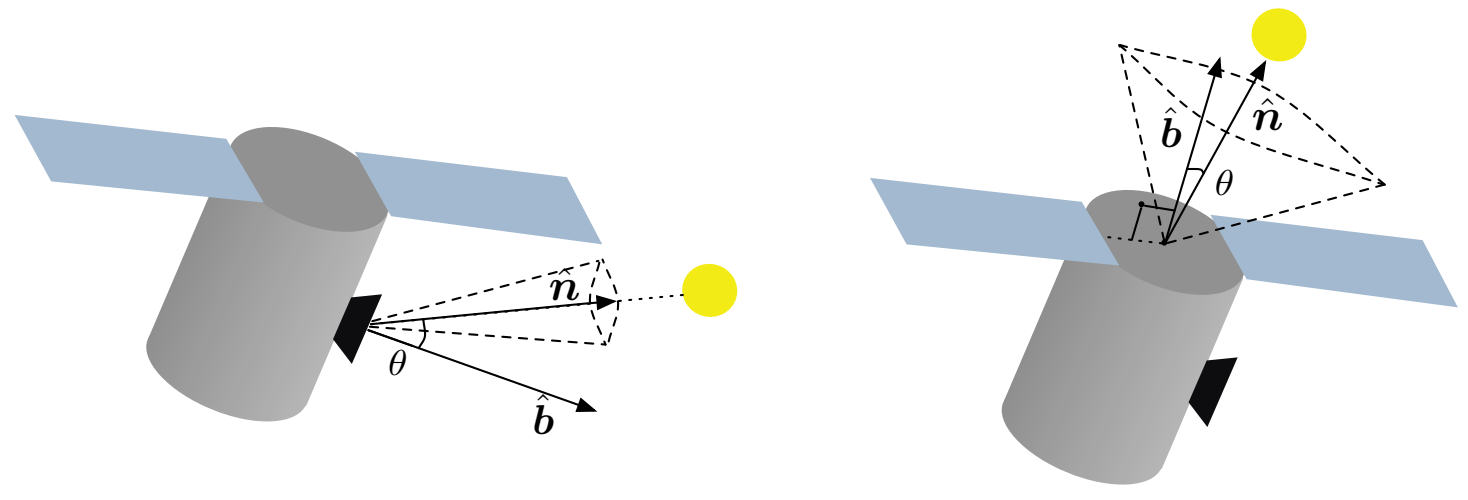

(a) Conic exclusion constraint. The spacecraft has (b) Conic inclusion constraint. The spacecraft has to slew keeping its sensitive optical instrument out to slew keeping the solar panels pointing somewhere of the sun-defined cone. inside the sun-defined cone.

Figure 3.1: Conic constraint geometry.

Conic constraints are thus written introducing the rotation matrix $[B N]$ as

$$
C_{[B N]}([B N])=\cos (\theta)-\cos \left(\theta_{\min }\right)=\hat{\boldsymbol{n}} \cdot \hat{\boldsymbol{b}}-\cos \left(\theta_{\min }\right)={ }^{\mathcal{N}} \hat{\boldsymbol{n}}^{T}[B N]^{T}{ }^{\mathcal{B}} \hat{\boldsymbol{b}}-\cos \left(\theta_{\min }\right)<0
$$

where the notation $C_{[B N]}$ indicates that the constraint is written as a function of the DCM.

Similarly, it might be desirable to maneuver while keeping a certain boresight vector $\hat{\boldsymbol{b}}$ inside a cone defined by $\hat{\boldsymbol{n}}$ and $\theta_{\min }$. For example, consider a maneuver that has to keep an antenna's main communication lobe inside a cone defined by a ground station. The mathematical condition is

$$
\hat{\boldsymbol{n}} \cdot \hat{\boldsymbol{b}}=\cos (\theta)>\cos \left(\theta_{\min }\right)
$$

Thus, the constraint can be written as a function of the DCM as

$$
C_{[B N]}([B N])=\cos (\theta)-\cos \left(\theta_{\min }\right)=\hat{\boldsymbol{n}} \cdot \hat{\boldsymbol{b}}-\cos \left(\theta_{\min }\right)={ }^{\mathcal{N}} \hat{\boldsymbol{n}}^{T}[B N]^{T}{ }^{\mathcal{B}} \hat{\boldsymbol{b}}-\cos \left(\theta_{\min }\right)>0
$$

It is important to notice that exclusion and inclusion zones are defined using the same constraint formulation; namely, through a function $C_{[B N]}([B N])$. From the conic constrained definition, the following inequality constraint must be true

$$
-2 \leq C_{[B N]}([B N]) \leq 2
$$


An expression for $\dot{C}_{[B N]}([B N])$ is readily computed using the transport theorem $[36]^{1}$ and the circular shift property of the triple product. If the derivatives are taken in the inertial frame under the hypothesis that $\hat{\boldsymbol{n}}$ is inertially constant and $\hat{\boldsymbol{b}}$ is body-fixed, then

$$
\dot{C}_{[B N]}([B N])=\frac{\mathcal{N}_{\mathrm{d}} \hat{\boldsymbol{n}}}{\mathrm{d} t} \cdot \hat{\boldsymbol{b}}+\hat{\boldsymbol{n}} \cdot \frac{\mathcal{N}_{\mathrm{d}} \hat{\boldsymbol{b}}}{\mathrm{d} t}=\hat{\boldsymbol{n}} \cdot\left(\boldsymbol{\omega}_{\mathcal{B} / \mathcal{N}} \times \hat{\boldsymbol{b}}\right)=(\hat{\boldsymbol{b}} \times \hat{\boldsymbol{n}}) \cdot \boldsymbol{\omega}_{\mathcal{B} / \mathcal{N}}=\left(\left[{ }^{\mathcal{B}} \tilde{\boldsymbol{b}}\right][B N]^{\mathcal{N}} \hat{\boldsymbol{n}}\right)^{T \mathcal{B}} \boldsymbol{\omega}_{\mathcal{B} / \mathcal{N}}
$$

Using Equation (2.3) the constraint and constraint rate expressions are rewritten as

$$
\begin{aligned}
& C_{[B N]}\left([B N]\left(\boldsymbol{\sigma}_{\mathcal{B} / \mathcal{N}}\right)\right)=C_{\boldsymbol{\sigma}}\left(\boldsymbol{\sigma}_{\mathcal{B} / \mathcal{N}}\right)={ }^{\mathcal{N}} \hat{\boldsymbol{n}}^{T}\left[B N\left(\boldsymbol{\sigma}_{\mathcal{B} / \mathcal{N}}\right)\right]^{T}{ }^{\mathcal{B}} \hat{\boldsymbol{b}}-\cos \left(\theta_{\min }\right) \\
& \dot{C}_{[B N]}\left([B N]\left(\boldsymbol{\sigma}_{\mathcal{B} / \mathcal{N}}\right)\right)=\dot{C}_{\boldsymbol{\sigma}}\left(\boldsymbol{\sigma}_{\mathcal{B} / \mathcal{N}}\right)=\left(\left[{ }^{\mathcal{B}} \tilde{\boldsymbol{b}}\right]\left[B N\left(\boldsymbol{\sigma}_{\mathcal{B} / \mathcal{N}}\right)\right]^{\mathcal{N}} \hat{\boldsymbol{n}}\right)^{T \mathcal{B}} \boldsymbol{\omega}_{\mathcal{B} / \mathcal{N}}
\end{aligned}
$$

It is also possible to compute the time derivative using the gradient and the kinematic differential equation given in 2.5

$$
\dot{C}_{[B N]}([B N])=\dot{C}_{\boldsymbol{\sigma}}\left(\boldsymbol{\sigma}_{\mathcal{B} / \mathcal{N}}\right)=\nabla C_{\boldsymbol{\sigma}}^{T}\left(\boldsymbol{\sigma}_{\mathcal{B} / \mathcal{N}}\right) \dot{\boldsymbol{\sigma}}_{\mathcal{B} / \mathcal{N}}=\frac{1}{4} \nabla C_{\boldsymbol{\sigma}}^{T}\left(\boldsymbol{\sigma}_{\mathcal{B} / \mathcal{N}}\right)\left[B\left(\boldsymbol{\sigma}_{\mathcal{B} / \mathcal{N}}\right)\right]^{\mathcal{B}} \boldsymbol{\omega}_{\mathcal{B} / \mathcal{N}}
$$

\footnotetext{
1 See Appendix A
} 


\section{Chapter 4}

\section{Attitude Constrained Steering Laws}

In this Chapter, the orientation constrained laws are derived for both the regulation and the tracking problem. Asymptotic stability of the control laws is discussed. After the derivations, numerical examples are given. Torque-bounded orientation-constrained laws are studied in detail in Chapter 5 .

\subsection{Constraint Overview}

Let there be $N_{\mathrm{E}}$ exclusion zones defined by continuous functions $C_{i}^{E}: \mathrm{SO}(3) \rightarrow \mathbb{R}$ and $N_{I}$ inclusion zones defined by continuous functions $C_{j}^{I}: \mathrm{SO}(3) \rightarrow \mathbb{R}$, which can be the functions $C_{[B N]}$ or $C_{\boldsymbol{\sigma}}$ described in Chapter 3 . Let $\mathcal{D}$ be a feasible attitude set such that

$$
\mathcal{D}=\left\{x \in \mathrm{SO}(3) / C_{i}^{E}(x)<0 \wedge C_{j}^{I}(x)>0\right\}
$$

The goal is to drive $\boldsymbol{\sigma}_{\mathcal{B} / \mathcal{R}}$ and $\boldsymbol{\omega}_{\mathcal{B} / \mathcal{R}}$ to zero while moving inside $\mathcal{D}$. The first necessary condition is that $[B N] \in \mathcal{D}$ for all possible times.

Barrier functions have been used to design control laws avoiding constraints [23, 28, 31, 47. In this thesis, logarithm barrier functions [23, 25, 47] are used to design Lyapunov functions 1 ] that converge to the reference while avoid piercing the static constraints.

\footnotetext{
${ }^{1}$ See Appendix $\mathrm{B}$ for a brief presentation on Lyapunov theory.
} 


\subsection{Attitude Constrained Regulation Steering Law}

In the regulation problem, the goal is to steer $\boldsymbol{\sigma}_{\mathcal{B} / \mathcal{N}}$ and $\boldsymbol{\omega}_{\mathcal{B} / \mathcal{N}}$ to zero. As in Chapter 2 , the problem is split into two parts: a steering law and a servo sub-system controlling angular velocity. Relative to the unconstrained law described in Chapter 2, the latter remains the same. The steering law has to be changed.

Consider the following Lyapunov candidate function $V: \mathcal{D} \rightarrow \mathbb{R}^{+}$

$$
V\left(\boldsymbol{\sigma}_{\mathcal{B} / \mathcal{N}}\right)=2 \ln \left(1+\boldsymbol{\sigma}_{\mathcal{B} / \mathcal{N}}^{T} \boldsymbol{\sigma}_{\mathcal{B} / \mathcal{N}}\right)\left[-\frac{1}{N_{E}} \sum_{i=1}^{N_{E}} \ln \left(-\frac{C_{i}^{E}\left(\boldsymbol{\sigma}_{\mathcal{B} / \mathcal{N}}\right)}{\alpha_{i}}\right)-\frac{1}{N_{I}} \sum_{j=1}^{N_{I}} \ln \left(\frac{C_{j}^{I}\left(\boldsymbol{\sigma}_{\mathcal{B} / \mathcal{N}}\right)}{\beta_{j}}\right)\right]
$$

The parameters $\alpha_{i}>0$ and $\beta_{j}>0$ can be chosen in several different ways with the only condition

$-C_{i}^{E}\left(\boldsymbol{\sigma}_{\mathcal{B} / \mathcal{N}}\right)<\alpha_{i}$ and $C_{j}^{I}\left(\boldsymbol{\sigma}_{\mathcal{B} / \mathcal{N}}\right)<\beta_{j} \forall \boldsymbol{\sigma}_{\mathcal{B} / \mathcal{N}} \in \mathcal{D}$. One possibility, based on Equation (3.7) is to pick $\alpha_{i}=\beta_{i}=2 e$. Therefore, the logarithm constraint terms will be between 1 and $+\infty$. Another possibility for $\alpha_{i}$ is discussed later.

The function has the following characteristics

(1) $V$ is continuously differentiable in $\mathcal{D}$.

(2) $V(\mathbf{0})=0$.

(3) $V\left(\boldsymbol{\sigma}_{\mathcal{B} / \mathcal{N}}\right)>0 \quad \forall \boldsymbol{\sigma}_{\mathcal{B} / \mathcal{N}} \in\{\mathcal{D}-\{\mathbf{0}\}\}$

Since $-C_{i}^{E}\left(\boldsymbol{\sigma}_{\mathcal{B} / \mathcal{N}}\right)<\alpha_{i}, C_{j}^{I}\left(\boldsymbol{\sigma}_{\mathcal{B} / \mathcal{N}}\right)<\beta_{j}$, and $-\ln (x)$ is a strictly decreasing function.

$$
\left[-\frac{1}{N_{E}} \sum_{i=1}^{N_{E}} \ln \left(-\frac{C_{i}^{E}\left(\boldsymbol{\sigma}_{\mathcal{B} / \mathcal{N}}\right)}{\alpha_{i}}\right)-\frac{1}{N_{I}} \sum_{j=1}^{N_{I}} \ln \left(\frac{C_{j}^{I}\left(\boldsymbol{\sigma}_{\mathcal{B} / \mathcal{N}}\right)}{\beta_{j}}\right)\right]>-\ln (1)-\ln (1)=0
$$

Given the fact that $\ln \left(1+\boldsymbol{\sigma}_{\mathcal{B} / \mathcal{N}}^{T} \boldsymbol{\sigma}_{\mathcal{B} / \mathcal{N}}\right)>0 \forall \boldsymbol{\sigma}_{\mathcal{B} / \mathcal{N}} \in\{\mathcal{D}-\{\mathbf{0}\}\}$, thus $V\left(\boldsymbol{\sigma}_{\mathcal{B} / \mathcal{N}}\right)>$ $0 \forall \boldsymbol{\sigma}_{\mathcal{B} / \mathcal{N}} \in\{\mathcal{D}-\{\mathbf{0}\}\}$.

(4) If $\alpha_{i}=\beta_{i}=2 e$

$$
\left[-\frac{1}{N_{E}} \sum_{i=1}^{N_{E}} \ln \left(-\frac{C_{i}^{E}\left(\boldsymbol{\sigma}_{\mathcal{B} / \mathcal{N}}\right)}{\alpha_{i}}\right)-\frac{1}{N_{I}} \sum_{j=1}^{N_{I}} \ln \left(\frac{C_{j}^{I}\left(\boldsymbol{\sigma}_{\mathcal{B} / \mathcal{N}}\right)}{\beta_{j}}\right)\right]>-\ln \left(\frac{1}{e}\right)-\ln \left(\frac{1}{e}\right)=2
$$


(5) $V\left(\boldsymbol{\sigma}_{\mathcal{B} / \mathcal{N}}\right) \rightarrow+\infty$ when $C_{i}^{E}\left(\boldsymbol{\sigma}_{\mathcal{B} / \mathcal{N}}\right) \rightarrow 0$ or $C_{i}^{I}\left(\boldsymbol{\sigma}_{\mathcal{B} / \mathcal{N}}\right) \rightarrow 0$

By conditions 1, 2, and 3, $V$ is a proper Lyapunov function[13]. Condition 5 ensures that the motion is constrained to $\mathcal{D} 2$

In order to derive a control law, the time derivative of $V$ is computed

$$
\begin{array}{r}
\dot{V}\left(\boldsymbol{\sigma}_{\mathcal{B} / \mathcal{N}}\right)=\frac{4 \boldsymbol{\sigma}_{\mathcal{B} / \mathcal{N}}^{T} \dot{\boldsymbol{\sigma}}_{\mathcal{B} / \mathcal{N}}}{\left(1+\boldsymbol{\sigma}_{\mathcal{B} / \mathcal{N}}^{T} \boldsymbol{\sigma}_{\mathcal{B} / \mathcal{N}}\right)}\left[-\frac{1}{N_{E}} \sum_{i=1}^{N_{E}} \ln \left(-\frac{C_{i}^{E}\left(\boldsymbol{\sigma}_{\mathcal{B} / \mathcal{N}}\right)}{\alpha_{i}}\right)-\frac{1}{N_{I}} \sum_{j=1}^{N_{I}} \ln \left(\frac{C_{j}^{I}\left(\boldsymbol{\sigma}_{\mathcal{B} / \mathcal{N}}\right)}{\beta_{j}}\right)\right]+ \\
2 \ln \left(1+\boldsymbol{\sigma}_{\mathcal{B} / \mathcal{N}}^{T} \boldsymbol{\sigma}_{\mathcal{B} / \mathcal{N}}\right)\left[-\frac{1}{N_{E}} \sum_{i=1}^{N_{E}} \frac{\dot{C}_{i}^{E}\left(\boldsymbol{\sigma}_{\mathcal{B} / \mathcal{N}}\right)}{C_{i}^{E}\left(\boldsymbol{\sigma}_{\mathcal{B} / \mathcal{N}}\right)}-\frac{1}{N_{I}} \sum_{j=1}^{N_{I}} \frac{\dot{C}_{j}^{I}\left(\boldsymbol{\sigma}_{\mathcal{B} / \mathcal{N}}\right)}{C_{j}^{I}\left(\boldsymbol{\sigma}_{\mathcal{B} / \mathcal{N}}\right)}\right]
\end{array}
$$

Using Equation 2.5 and the fact ${ }^{3}$ that $\boldsymbol{\sigma}_{\mathcal{B} / \mathcal{N}}^{T}\left[\tilde{\boldsymbol{\sigma}}_{\mathcal{B} / \mathcal{N}}\right]=0$

$$
\begin{aligned}
\dot{V}\left(\boldsymbol{\sigma}_{\mathcal{B} / \mathcal{N}}\right)=\boldsymbol{\sigma}_{\mathcal{B} / \mathcal{N}}^{T}{ }^{\mathcal{B}} \boldsymbol{\omega}_{\mathcal{B} / \mathcal{N}} & {\left[-\frac{1}{N_{E}} \sum_{i=1}^{N_{E}} \ln \left(-\frac{C_{i}^{E}\left(\boldsymbol{\sigma}_{\mathcal{B} / \mathcal{N}}\right)}{\alpha_{i}}\right)-\frac{1}{N_{I}} \sum_{j=1}^{N_{I}} \ln \left(\frac{C_{j}^{I}\left(\boldsymbol{\sigma}_{\mathcal{B} / \mathcal{N}}\right)}{\beta_{j}}\right)\right]+} \\
2 & \ln \left(1+\boldsymbol{\sigma}_{\mathcal{B} / \mathcal{N}}^{T} \boldsymbol{\sigma}_{\mathcal{B} / \mathcal{N}}\right)\left[-\frac{1}{N_{E}} \sum_{i=1}^{N_{E}} \frac{\dot{C}_{i}^{E}\left(\boldsymbol{\sigma}_{\mathcal{B} / \mathcal{N})}\right.}{C_{i}^{E}\left(\boldsymbol{\sigma}_{\mathcal{B} / \mathcal{N}}\right)}-\frac{1}{N_{I}} \sum_{j=1}^{N_{I}} \frac{\dot{C}_{j}^{I}\left(\boldsymbol{\sigma}_{\mathcal{B} / \mathcal{N})}\right)}{C_{j}^{I}\left(\boldsymbol{\sigma}_{\mathcal{B} / \mathcal{N}}\right)}\right]
\end{aligned}
$$

Using Equation (3.8) and re-arranging

$$
\begin{gathered}
\dot{V}\left(\boldsymbol{\sigma}_{\mathcal{B} / \mathcal{N}}\right)=\left(\boldsymbol{\sigma}_{\mathcal{B} / \mathcal{N}}^{T}\left[-\frac{1}{N_{E}} \sum_{i=1}^{N_{E}} \ln \left(-\frac{C_{i}^{E}\left(\boldsymbol{\sigma}_{\mathcal{B} / \mathcal{N}}\right)}{\alpha_{i}}\right)-\frac{1}{N_{I}} \sum_{j=1}^{N_{I}} \ln \left(\frac{C_{j}^{I}\left(\boldsymbol{\sigma}_{\mathcal{B} / \mathcal{N}}\right)}{\beta_{j}}\right)\right]+\right. \\
\left.2 \ln \left(1+\boldsymbol{\sigma}_{\mathcal{B} / \mathcal{N}}^{T} \boldsymbol{\sigma}_{\mathcal{B} / \mathcal{N}}\right)\left[-\frac{1}{N_{E}} \sum_{i=1}^{N_{E}} \frac{\left(\left[{ }^{\mathcal{B}} \tilde{\boldsymbol{b}}\right][B N]^{\mathcal{N}} \hat{\boldsymbol{n}}\right)^{T}}{C_{j}^{E}\left(\boldsymbol{\sigma}_{\mathcal{B} / \mathcal{N}}\right)}-\frac{1}{N_{I}} \sum_{j=1}^{N_{I}} \frac{\left(\left[{ }^{\mathcal{B}} \tilde{\boldsymbol{b}}\right][B N]^{\mathcal{N}} \hat{\boldsymbol{n}}\right)^{T}}{C_{j}^{I}\left(\boldsymbol{\sigma}_{\mathcal{B} / \mathcal{N}}\right)}\right]\right){ }^{\mathcal{B}} \boldsymbol{\omega}_{\mathcal{B} / \mathcal{N}}
\end{gathered}
$$

Letting $\boldsymbol{v}_{R}$ be

$$
\begin{aligned}
\boldsymbol{v}_{R}=\left(\boldsymbol{\sigma}_{\mathcal{B} / \mathcal{N}}[\right. & \left.-\frac{1}{N_{E}} \sum_{i=1}^{N_{E}} \ln \left(-\frac{C_{i}^{E}\left(\boldsymbol{\sigma}_{\mathcal{B} / \mathcal{N}}\right)}{\alpha_{i}}\right)-\frac{1}{N_{I}} \sum_{j=1}^{N_{I}} \ln \left(\frac{C_{j}^{I}\left(\boldsymbol{\sigma}_{\mathcal{B} / \mathcal{N}}\right)}{\beta_{j}}\right)\right]+ \\
& \left.2 \ln \left(1+\boldsymbol{\sigma}_{\mathcal{B} / \mathcal{N}}^{T} \boldsymbol{\sigma}_{\mathcal{B} / \mathcal{N}}\right)\left[-\frac{1}{N_{E}} \sum_{i=1}^{N_{E}} \frac{\left(\left[{ }^{\mathcal{B}} \tilde{\boldsymbol{b}}\right][B N]^{\mathcal{N}} \hat{\boldsymbol{n}}\right)}{C_{i}^{E}\left(\boldsymbol{\sigma}_{\mathcal{B} / \mathcal{N}}\right)}-\frac{1}{N_{I}} \sum_{j=1}^{N_{I}} \frac{\left(\left[{ }^{\mathcal{B}} \tilde{\boldsymbol{b}}\right][B N]^{\mathcal{N}} \hat{\boldsymbol{n}}\right)}{C_{j}^{I}\left(\boldsymbol{\sigma}_{\mathcal{B} / \mathcal{N}}\right)}\right]\right)
\end{aligned}
$$

it is possible to write

$$
\dot{V}\left(\boldsymbol{\sigma}_{\mathcal{B} / \mathcal{N}}\right)=\boldsymbol{v}_{R}^{T \mathcal{B}} \boldsymbol{\omega}_{\mathcal{B} / \mathcal{N}}
$$

\footnotetext{
${ }^{2}$ See Appendix $\mathrm{B}$

${ }^{3}$ See Appendix $\bar{A}$ for details.
} 
It is important to notice that $\boldsymbol{v}_{R}=0$ whenever $\boldsymbol{\sigma}_{\mathcal{B} / \mathcal{N}}=\mathbf{0}$.

Choosing

$$
{ }^{\mathcal{B}} \boldsymbol{\omega}_{\mathcal{B}^{*} / \mathcal{N}}=-\boldsymbol{f}\left(\boldsymbol{v}_{R}\right)
$$

where $\boldsymbol{f}$ is given by Equation 2.16

$$
\dot{V}\left(\boldsymbol{\sigma}_{\mathcal{B} / \mathcal{N}}\right)=-\boldsymbol{v}_{R}^{T} \boldsymbol{f}\left(\boldsymbol{v}_{R}\right) \leq 0
$$

Notice that the control law given in Equation 4.10 is rate-bounded.

According to Lyapunov's direct method[13], since $\dot{V}$ is negative semi-definite, the steering law given by Equation 4.10 is stable.

For asymptotic stability, $\dot{V}$ has to be negative definite. In other words, $\boldsymbol{v}_{R}$ has to be $\mathbf{0}$ only when $\boldsymbol{\sigma}_{\mathcal{B} / \mathcal{N}}=\mathbf{0}$. This occurs almost everywhere in every single case, but there might be certain orientations in some specific problem conditions in which $\boldsymbol{v}_{R}=\mathbf{0}$ with $\boldsymbol{\sigma}_{\mathcal{B} / \mathcal{N}} \neq \mathbf{0}$. This situation is studied in Section 4.4 .

\subsection{Attitude Constrained Tracking Steering Law}

In the tracking problem, the goal is to steer $\boldsymbol{\sigma}_{\mathcal{B} / \mathcal{R}}$ and $\boldsymbol{\omega}_{\mathcal{B} / \mathcal{R}}$ to zero. As in Chapter 2 , the problem is split into two parts: a steering law and a servo sub-system, which controls angular velocity. Relative to the unconstrained law described in Chapter 2, the latter remains the same. The steering law has to be changed.

Consider the following Lyapunov candidate function $V: \mathcal{D} \rightarrow \mathbb{R}^{+}$

$$
V\left(\boldsymbol{\sigma}_{\mathcal{B} / \mathcal{R}}\right)=2 \ln \left(1+\boldsymbol{\sigma}_{\mathcal{B} / \mathcal{R}}^{T} \boldsymbol{\sigma}_{\mathcal{B} / \mathcal{R}}\right)\left[-\frac{1}{N_{E}} \sum_{i=1}^{N_{E}} \ln \left(-\frac{C_{i}^{E}\left(\boldsymbol{\sigma}_{\mathcal{B} / \mathcal{N}}\right)}{\alpha_{i}}\right)-\frac{1}{N_{I}} \sum_{j=1}^{N_{I}} \ln \left(\frac{C_{j}^{I}\left(\boldsymbol{\sigma}_{\mathcal{B} / \mathcal{N}}\right)}{\beta_{j}}\right)\right]
$$

This Lyapunov function is similar to the one studied in Section 4.2. However, the problem is more complex since, in this case, $V$ depends on $\boldsymbol{\sigma}_{\mathcal{B} / \mathcal{R}}$ and $\boldsymbol{\sigma}_{\mathcal{B} / \mathcal{N}}$ simultaneously.

If the parameters $\alpha_{i}>0$ and $\beta_{j}>0$ are chosen as in Section 4.2 , $V$ will be a proper Lyapunov candidate function and will have the properties already studied in that section. 
Its time derivative is given by

$$
\begin{array}{r}
\dot{V}\left(\boldsymbol{\sigma}_{\mathcal{B} / \mathcal{R}}\right)=\frac{4 \boldsymbol{\sigma}_{\mathcal{B} / \mathcal{R}}^{T} \dot{\boldsymbol{\sigma}}_{\mathcal{B} / \mathcal{R}}}{\left(1+\boldsymbol{\sigma}_{\mathcal{B} / \mathcal{R}}^{T} \boldsymbol{\sigma}_{\mathcal{B} / \mathcal{R}}\right)}\left[-\frac{1}{N_{E}} \sum_{i=1}^{N_{E}} \ln \left(-\frac{C_{i}^{E}\left(\boldsymbol{\sigma}_{\mathcal{B} / \mathcal{N}}\right)}{\alpha_{i}}\right)-\frac{1}{N_{I}} \sum_{j=1}^{N_{I}} \ln \left(\frac{C_{j}^{I}\left(\boldsymbol{\sigma}_{\mathcal{B} / \mathcal{N}}\right)}{\beta_{j}}\right)\right]+ \\
2 \ln \left(1+\boldsymbol{\sigma}_{\mathcal{B} / \mathcal{R}}^{T} \boldsymbol{\sigma}_{\mathcal{B} / \mathcal{R}}\right)\left[-\frac{1}{N_{E}} \sum_{i=1}^{N_{E}} \frac{\dot{C}_{i}^{E}\left(\boldsymbol{\sigma}_{\mathcal{B} / \mathcal{N}}\right)}{C_{i}^{E}\left(\boldsymbol{\sigma}_{\mathcal{B} / \mathcal{N}}\right)}-\frac{1}{N_{I}} \sum_{j=1}^{N_{I}} \frac{\dot{C}_{j}^{I}\left(\boldsymbol{\sigma}_{\mathcal{B} / \mathcal{N}}\right)}{C_{j}^{I}\left(\boldsymbol{\sigma}_{\mathcal{B} / \mathcal{N}}\right)}\right]
\end{array}
$$

Using Equation 2.5 and the fact ${ }^{4}$ that $\boldsymbol{\sigma}_{\mathcal{B} / \mathcal{R}}^{T}\left[\tilde{\boldsymbol{\sigma}}_{\mathcal{B} / \mathcal{R}}\right]=0$

$$
\begin{aligned}
\dot{V}\left(\boldsymbol{\sigma}_{\mathcal{B} / \mathcal{R}}\right)=\boldsymbol{\sigma}_{\mathcal{B} / \mathcal{R}}^{T} \boldsymbol{\omega}_{\mathcal{B} / \mathcal{R}} & {\left[-\frac{1}{N_{E}} \sum_{i=1}^{N_{E}} \ln \left(-\frac{C_{i}^{E}\left(\boldsymbol{\sigma}_{\mathcal{B} / \mathcal{N}}\right)}{\alpha_{i}}\right)-\frac{1}{N_{I}} \sum_{j=1}^{N_{I}} \ln \left(\frac{C_{j}^{I}\left(\boldsymbol{\sigma}_{\mathcal{B} / \mathcal{N}}\right)}{\beta_{j}}\right)\right]+} \\
2 & \ln \left(1+\boldsymbol{\sigma}_{\mathcal{B} / \mathcal{R}}^{T} \boldsymbol{\sigma}_{\mathcal{B} / \mathcal{R}}\right)\left[-\frac{1}{N_{E}} \sum_{i=1}^{N_{E}} \frac{\dot{C}_{i}^{E}\left(\boldsymbol{\sigma}_{\mathcal{B} / \mathcal{N}}\right)}{C_{i}^{E}\left(\boldsymbol{\sigma}_{\mathcal{B} / \mathcal{N}}\right)}-\frac{1}{N_{I}} \sum_{j=1}^{N_{I}} \frac{\dot{C}_{j}^{I}\left(\boldsymbol{\sigma}_{\mathcal{B} / \mathcal{N}}\right)}{C_{j}^{I}\left(\boldsymbol{\sigma}_{\mathcal{B} / \mathcal{N}}\right)}\right]
\end{aligned}
$$

Plugging Equation (3.8),

$$
\begin{gathered}
\dot{V}\left(\boldsymbol{\sigma}_{\mathcal{B} / \mathcal{R}}\right)=\boldsymbol{\sigma}_{\mathcal{B} / \mathcal{R}}^{T}{ }^{\mathcal{B}} \boldsymbol{\omega}_{\mathcal{B} / \mathcal{R}}\left[-\frac{1}{N_{E}} \sum_{i=1}^{N_{E}} \ln \left(-\frac{C_{i}^{E}\left(\boldsymbol{\sigma}_{\mathcal{B} / \mathcal{N}}\right)}{\alpha_{i}}\right)-\frac{1}{N_{I}} \sum_{j=1}^{N_{I}} \ln \left(\frac{C_{j}^{I}\left(\boldsymbol{\sigma}_{\mathcal{B} / \mathcal{N}}\right)}{\beta_{j}}\right)\right]+ \\
2 \ln \left(1+\boldsymbol{\sigma}_{\mathcal{B} / \mathcal{R}}^{T} \boldsymbol{\sigma}_{\mathcal{B} / \mathcal{R}}\right)\left[-\frac{1}{N_{E}} \sum_{i=1}^{N_{E}} \frac{\left(\left[{ }^{\mathcal{B}} \tilde{\boldsymbol{b}}\right][B N]^{\mathcal{N}} \hat{\boldsymbol{n}}\right)^{T}}{C_{j}^{E}\left(\boldsymbol{\sigma}_{\mathcal{B} / \mathcal{N}}\right)}-\frac{1}{N_{I}} \sum_{j=1}^{N_{I}} \frac{\left(\left[{ }^{\mathcal{B}} \tilde{\boldsymbol{b}}\right][B N]^{\mathcal{N}} \hat{\boldsymbol{n}}\right)^{T}}{C_{j}^{I}\left(\boldsymbol{\sigma}_{\mathcal{B} / \mathcal{N}}\right)}\right]{ }^{\mathcal{B}} \boldsymbol{\omega}_{\mathcal{B} / \mathcal{N}}
\end{gathered}
$$

Since $\boldsymbol{\omega}_{\mathcal{B} / \mathcal{N}}=\boldsymbol{\omega}_{\mathcal{B} / \mathcal{R}}+\boldsymbol{\omega}_{\mathcal{R} / \mathcal{N}}$

$$
\begin{gathered}
\dot{V}\left(\boldsymbol{\sigma}_{\mathcal{B} / \mathcal{R}}\right)=\left(\boldsymbol{\sigma}_{\mathcal{B} / \mathcal{R}}^{T}\left[\frac{1}{N_{E}} \sum_{i=1}^{N_{E}}-\ln \left(-\frac{C_{i}^{E}\left(\boldsymbol{\sigma}_{\mathcal{B} / \mathcal{N}}\right)}{\alpha_{i}}\right)-\frac{1}{N_{I}} \sum_{j=1}^{N_{I}} \ln \left(\frac{C_{j}^{I}\left(\boldsymbol{\sigma}_{\mathcal{B} / \mathcal{N}}\right)}{\beta_{j}}\right)\right]+\right. \\
\left.2 \ln \left(1+\boldsymbol{\sigma}_{\mathcal{B} / \mathcal{R}}^{T} \boldsymbol{\sigma}_{\mathcal{B} / \mathcal{R}}\right)\left[-\frac{1}{N_{E}} \sum_{i=1}^{N_{E}} \frac{\left(\left[{ }^{\mathcal{B}} \tilde{\boldsymbol{b}}\right][B N]^{\mathcal{N}} \hat{\boldsymbol{n}}\right)^{T}}{C_{j}^{E}\left(\boldsymbol{\sigma}_{\mathcal{B} / \mathcal{N}}\right)}-\frac{1}{N_{I}} \sum_{j=1}^{N_{I}} \frac{\left(\left[{ }^{\mathcal{B}} \tilde{\boldsymbol{b}}\right][B N]^{\mathcal{N}} \hat{\boldsymbol{n}}\right)^{T}}{C_{j}^{I}\left(\boldsymbol{\sigma}_{\mathcal{B} / \mathcal{N}}\right)}\right]\right){ }^{\mathcal{B}} \boldsymbol{\omega}_{\mathcal{B} / \mathcal{R}}+ \\
2 \ln \left(1+\boldsymbol{\sigma}_{\mathcal{B} / \mathcal{R}}^{T} \boldsymbol{\sigma}_{\mathcal{B} / \mathcal{R}}\right)\left[-\frac{1}{N_{E}} \sum_{i=1}^{N_{E}} \frac{\left.\left({ }^{\mathcal{B}} \tilde{\boldsymbol{b}}\right][B N]^{\mathcal{N}} \hat{\boldsymbol{n}}\right)^{T}}{C_{j}^{E}\left(\boldsymbol{\sigma}_{\mathcal{B} / \mathcal{N}}\right)}-\frac{1}{N_{I}} \sum_{j=1}^{N_{I}} \frac{\left(\left[{ }^{\mathcal{B}} \tilde{\boldsymbol{b}}\right][B N]^{\mathcal{N}} \hat{\boldsymbol{n}}\right)^{T}}{C_{j}^{I}\left(\boldsymbol{\sigma}_{\mathcal{B} / \mathcal{N}}\right)}\right]{ }^{\mathcal{B}} \boldsymbol{\omega}_{\mathcal{R} / \mathcal{N}}
\end{gathered}
$$

Let $\boldsymbol{v}_{T}$ and $\boldsymbol{u}_{T}$ be such that

$$
\begin{aligned}
& \boldsymbol{u}_{T}=2 \ln \left(1+\boldsymbol{\sigma}_{\mathcal{B} / \mathcal{R}}^{T} \boldsymbol{\sigma}_{\mathcal{B} / \mathcal{R}}\right)\left[-\frac{1}{N_{E}} \sum_{i=1}^{N_{E}} \frac{\left(\left[{ }^{\mathcal{B}} \tilde{\boldsymbol{b}}\right][B N]^{\mathcal{N}} \hat{\boldsymbol{n}}\right)}{C_{j}^{E}\left(\boldsymbol{\sigma}_{\mathcal{B} / \mathcal{N}}\right)}-\frac{1}{N_{I}} \sum_{j=1}^{N_{I}} \frac{\left(\left[{ }^{\mathcal{B}} \tilde{\boldsymbol{b}}\right][B N]^{\mathcal{N}} \hat{\boldsymbol{n}}\right)}{C_{j}^{I}\left(\boldsymbol{\sigma}_{\mathcal{B} / \mathcal{N}}\right)}\right] \\
& \boldsymbol{v}_{T}=\boldsymbol{\sigma}_{\mathcal{B} / \mathcal{R}}\left[\frac{1}{N_{E}} \sum_{i=1}^{N_{E}}-\ln \left(-\frac{C_{i}^{E}\left(\boldsymbol{\sigma}_{\mathcal{B} / \mathcal{N}}\right)}{\alpha_{i}}\right)-\frac{1}{N_{I}} \sum_{j=1}^{N_{I}} \ln \left(\frac{C_{j}^{I}\left(\boldsymbol{\sigma}_{\mathcal{B} / \mathcal{N}}\right)}{\beta_{j}}\right)\right]+\boldsymbol{u}_{T}
\end{aligned}
$$

\footnotetext{
${ }^{4}$ See Appendix A for details.
} 
It is important to notice that $\boldsymbol{v}_{T}=\boldsymbol{u}_{T}=\mathbf{0}$ whenever $\boldsymbol{\sigma}_{\mathcal{B} / \mathcal{R}}=\mathbf{0}$.

Then

$$
\dot{V}\left(\boldsymbol{\sigma}_{\mathcal{B} / \mathcal{R}}\right)=\boldsymbol{v}_{T}^{T \mathcal{B}} \boldsymbol{\omega}_{\mathcal{B} / \mathcal{R}}+\boldsymbol{u}_{T}^{T \mathcal{B}} \boldsymbol{\omega}_{\mathcal{R} / \mathcal{N}}
$$

Choosing

$$
{ }^{\mathcal{B}} \boldsymbol{\omega}_{\mathcal{B}^{*} / \mathcal{R}}=-\boldsymbol{f}\left(\boldsymbol{v}_{T}\right)-\frac{\boldsymbol{v}_{T} \boldsymbol{u}_{T}^{T}}{\boldsymbol{v}_{T}^{T} \boldsymbol{v}_{T}} \boldsymbol{\omega}_{\mathcal{R} / \mathcal{N}}
$$

where $\boldsymbol{f}$ is given by Equation 2.16

$$
\dot{V}\left(\boldsymbol{\sigma}_{\mathcal{B} / \mathcal{R}}\right)=-\boldsymbol{v}_{T}^{T} \boldsymbol{f}\left(\boldsymbol{v}_{T}\right) \leq 0
$$

Since $\boldsymbol{\sigma}_{\mathcal{B} / \mathcal{R}}=\mathbf{0}$ implies $\boldsymbol{v}_{T}=\boldsymbol{u}_{T}=\mathbf{0}$, the second term in Equation 4.20 contains a $\frac{0}{0}$ indetermination. However, $\ln \left(1+\boldsymbol{\sigma}_{\mathcal{B} / \mathcal{R}}^{T} \boldsymbol{\sigma}_{\mathcal{B} / \mathcal{R}}\right) \rightarrow \boldsymbol{\sigma}_{\mathcal{B} / \mathcal{R}}^{T} \boldsymbol{\sigma}_{\mathcal{B} / \mathcal{R}}$ when $\boldsymbol{\sigma}_{\mathcal{B} / \mathcal{R}} \rightarrow 0$. Thus it is possible to write

$$
\begin{aligned}
& \boldsymbol{u}_{T} \underset{\sigma_{\mathcal{B} / \mathcal{R}} \rightarrow 0}{\longrightarrow} \boldsymbol{\sigma}_{\mathcal{B} / \mathcal{R}}^{T} \boldsymbol{\sigma}_{\mathcal{B} / \mathcal{R}} \boldsymbol{a} \\
& \boldsymbol{v}_{T} \underset{\sigma_{\mathcal{B} / \mathcal{R}} \rightarrow 0}{\longrightarrow} \beta \boldsymbol{\sigma}_{\mathcal{B} / \mathcal{R}}+\boldsymbol{\sigma}_{\mathcal{B} / \mathcal{R}}^{T} \boldsymbol{\sigma}_{\mathcal{B} / \mathcal{R}} \boldsymbol{a}
\end{aligned}
$$

The vector $\boldsymbol{a}$ and the scalar $\beta$ do not depend on the attitude error.

Therefore

$$
\begin{aligned}
& \boldsymbol{v}_{T}^{T} \boldsymbol{v}_{T} \underset{\boldsymbol{\sigma}_{\mathcal{B} / \mathcal{R}} \rightarrow 0}{\longrightarrow} \boldsymbol{\sigma}_{\mathcal{B} / \mathcal{R}}^{T} \boldsymbol{\sigma}_{\mathcal{B} / \mathcal{R}} \beta^{2}+\left(\boldsymbol{\sigma}_{\mathcal{B} / \mathcal{R}}^{T} \boldsymbol{\sigma}_{\mathcal{B} / \mathcal{R}}\right)^{2} \boldsymbol{a}^{T} \boldsymbol{a}+2 \beta \boldsymbol{\sigma}_{\mathcal{B} / \mathcal{R}}^{T} \boldsymbol{\sigma}_{\mathcal{B} / \mathcal{R}} \boldsymbol{\sigma}_{\mathcal{B} / \mathcal{R}}^{T} \boldsymbol{a} \\
& \boldsymbol{v}_{T} \boldsymbol{u}_{T}^{T \mathcal{B}} \boldsymbol{\omega}_{\mathcal{R} / \mathcal{N}} \underset{\boldsymbol{\sigma}_{\mathcal{B} / \mathcal{R}} \rightarrow 0}{\longrightarrow} \beta \boldsymbol{\sigma}_{\mathcal{B} / \mathcal{R}} \boldsymbol{\sigma}_{\mathcal{B} / \mathcal{R}}^{T} \boldsymbol{\sigma}_{\mathcal{B} / \mathcal{R}} \boldsymbol{a}^{T \mathcal{B}} \boldsymbol{\omega}_{\mathcal{R} / \mathcal{N}}+\left(\boldsymbol{\sigma}_{\mathcal{B} / \mathcal{R}}^{T} \boldsymbol{\sigma}_{\mathcal{B} / \mathcal{R}}\right)^{2} \boldsymbol{a} \boldsymbol{a}^{T \mathcal{B}} \boldsymbol{\omega}_{\mathcal{R} / \mathcal{N}}
\end{aligned}
$$

When $\boldsymbol{\sigma}_{\mathcal{B} / \mathcal{R}} \rightarrow 0$, the expressions can be further approximated by dropping higher order terms

$$
\begin{aligned}
& \boldsymbol{v}_{T}^{T} \boldsymbol{v}_{T} \underset{\boldsymbol{\sigma}_{\mathcal{B} / \mathcal{R}} \rightarrow 0}{\longrightarrow} \boldsymbol{\sigma}_{\mathcal{B} / \mathcal{R}}^{T} \boldsymbol{\sigma}_{\mathcal{B} / \mathcal{R}} \beta^{2} \\
& \boldsymbol{v}_{T} \boldsymbol{u}_{T}^{T \mathcal{B}} \boldsymbol{\omega}_{\mathcal{R} / \mathcal{N}} \underset{\boldsymbol{\sigma}_{\mathcal{B} / \mathcal{R}} \rightarrow 0}{\longrightarrow} \beta \boldsymbol{\sigma}_{\mathcal{B} / \mathcal{R}} \boldsymbol{\sigma}_{\mathcal{B} / \mathcal{R}}^{T} \boldsymbol{\sigma}_{\mathcal{B} / \mathcal{R}} \boldsymbol{a}^{T \mathcal{B}} \boldsymbol{\omega}_{\mathcal{R} / \mathcal{N}}
\end{aligned}
$$

Hence

$$
\frac{\boldsymbol{v}_{T} \boldsymbol{u}_{T}^{T} \mathcal{B}_{T}}{\boldsymbol{v}_{T}^{T} \boldsymbol{v}_{T}} \boldsymbol{\omega}_{\mathcal{R} / \mathcal{N}} \underset{\boldsymbol{\sigma}_{\mathcal{B} / \mathcal{R}} \rightarrow 0}{\longrightarrow} \frac{\beta \boldsymbol{\sigma}_{\mathcal{B} / \mathcal{R}} \boldsymbol{\sigma}_{\mathcal{B} / \mathcal{R}}^{T} \boldsymbol{\sigma}_{\mathcal{B} / \mathcal{R}} \boldsymbol{a}^{T \mathcal{B}} \boldsymbol{\omega}_{\mathcal{R} / \mathcal{N}}}{\boldsymbol{\sigma}_{\mathcal{B} / \mathcal{R}}^{T} \boldsymbol{\sigma}_{\mathcal{B} / \mathcal{R}} \beta^{2}}=\frac{\boldsymbol{\sigma}_{\mathcal{B} / \mathcal{R}} \boldsymbol{a}^{T \mathcal{B}} \boldsymbol{\omega}_{\mathcal{R} / \mathcal{N}}}{\beta}
$$




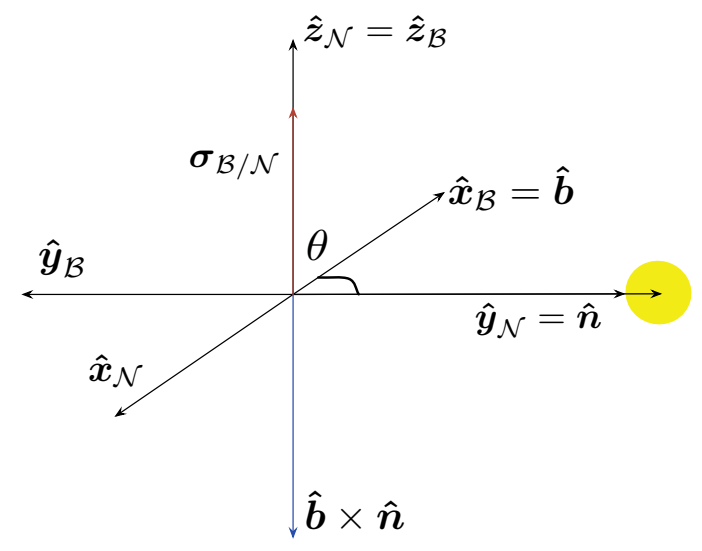

Figure 4.1: With perfect symmetry $\boldsymbol{v}_{R}$ can be $\mathbf{0}$.

This derivation solves the $\frac{0}{0}$ indetermination and shows that the second term can be modeled either by a linear function of the attitude error or by $\mathbf{0}$ when $\boldsymbol{\sigma}_{\mathcal{B} / \mathcal{R}}$ is small.

Another important remark is that, in contrast with the regulation law in Equation 4.10, the tracking law given by Equation (4.20) is not necesarilly rate-bounded.

If the vector $\boldsymbol{v}_{T}$ is zero when $\boldsymbol{\sigma}_{\mathcal{B} / \mathcal{R}} \neq \mathbf{0}$, the controlability is lost. This situation is studied in the following section.

\subsection{Convergence Analysis}

It has been shown that the laws given by Equations (4.10) and 4.20) are Lyapunov stable but not necessarily asymptotically stable. Consider, for example, the special case in which $\boldsymbol{v}_{T}=\mathbf{0}$ and $\boldsymbol{\sigma}_{\mathcal{B} / \mathcal{R}} \neq \mathbf{0}$. Fortunately, it turns out that this occurs only with very specific symmetry conditions.

To understand the geometric conditions that lead to this situation, consider the following regulation problem $\left(\boldsymbol{\sigma}_{\mathcal{R} / \mathcal{N}}=\mathbf{0}\right)$, depicted in Figure 4.1. Let there only be one exclusion condition, given by $\hat{\boldsymbol{n}}=\hat{\boldsymbol{y}}_{N}$. The boresight vector is in the body $\hat{\boldsymbol{x}}$ direction: $\hat{\boldsymbol{b}}=\hat{\boldsymbol{x}}_{B}$. In this qualitative description, the angle $\theta_{\min }$ is not relevant. The initial attitude is a rotation of $180^{\circ}$ about $\hat{\boldsymbol{z}}_{N}$. Thus $\mathrm{s}^{5} \boldsymbol{\sigma}_{\mathcal{B} / \mathcal{N}}=\tan \left(180^{\circ} / 4\right) \hat{\boldsymbol{z}}_{N}$.

\footnotetext{
${ }^{5}$ See Section 2.1 .
} 
The vector $\boldsymbol{v}_{R}$ is

$$
\boldsymbol{v}_{R}=-\boldsymbol{\sigma}_{\mathcal{B} / \mathcal{N}} \ln \left(-\frac{C_{1}^{E}\left(\boldsymbol{\sigma}_{\mathcal{B} / \mathcal{N}}\right)}{\alpha_{1}}\right)-2 \ln \left(1+\boldsymbol{\sigma}_{\mathcal{B} / \mathcal{N}}^{T} \boldsymbol{\sigma}_{\mathcal{B} / \mathcal{N}}\right) \frac{\left(\left[{ }^{\mathcal{B}} \tilde{\boldsymbol{b}}\right][B N]^{\mathcal{N}} \hat{\boldsymbol{n}}\right)}{C_{1}^{E}\left(\boldsymbol{\sigma}_{\mathcal{B} / \mathcal{N}}\right)}
$$

A necessary (but not sufficient) condition for $\boldsymbol{v}_{R}$ to be zero with a non-zero $\boldsymbol{\sigma}_{\mathcal{B} / \mathcal{N}}$ is $\boldsymbol{\sigma}_{\mathcal{B} / \mathcal{N}}$ and $\left[{ }^{\mathcal{B}} \tilde{\boldsymbol{b}}\right][B N]^{\mathcal{N}} \hat{\boldsymbol{n}}$ to be anti-parallel. The latter is simply $\hat{\boldsymbol{b}} \times \hat{\boldsymbol{n}}$. In this particular case, that situation is possible since the attitude is a rotation about the $\hat{\boldsymbol{z}}_{N}$ axis and $\hat{\boldsymbol{b}} \times \hat{\boldsymbol{n}}$ is in the same direction. These saddle points can occur whenever the problem has one of such perfect symmetries. If the conic constraint is slightly tilted, the symmetry is broken, and no saddle point is reached for that attitude initial condition.

In practice, due to perturbations and numerical noise, saddle points occur very rarely. However, $\boldsymbol{v}_{R}\left(\right.$ or $\boldsymbol{v}_{T}$ ) can become arbitrarily small.

It should be clear that these saddle-points are zero-measure sets and do not affect asymptotic stability as long as there exists some mechanism to escape from them. In other words, the control laws are asymptotically stable anywhere else.

A heuristic solution to avoid these saddle points is to detect whenever $\boldsymbol{v}_{R}$ (or $\boldsymbol{v}_{T}$ ) is small while $\boldsymbol{\sigma}_{\mathcal{B} / \mathcal{R}}$ is not and apply a very small push to the spacecraft in any direction orthogonal to $\boldsymbol{\sigma}_{\mathcal{B} / \mathcal{R}}$ in order to break the symmetry. The heuristic algorithm is shown as Algorithm 1 , $\boldsymbol{\sigma}_{\mathcal{B} / \mathcal{R}}^{\perp}$ is any orthogonal vector to $\boldsymbol{\sigma}_{\mathcal{B} / \mathcal{R}}$. The scalar algorithm parameter $\gamma$ is a small number to be chosen.

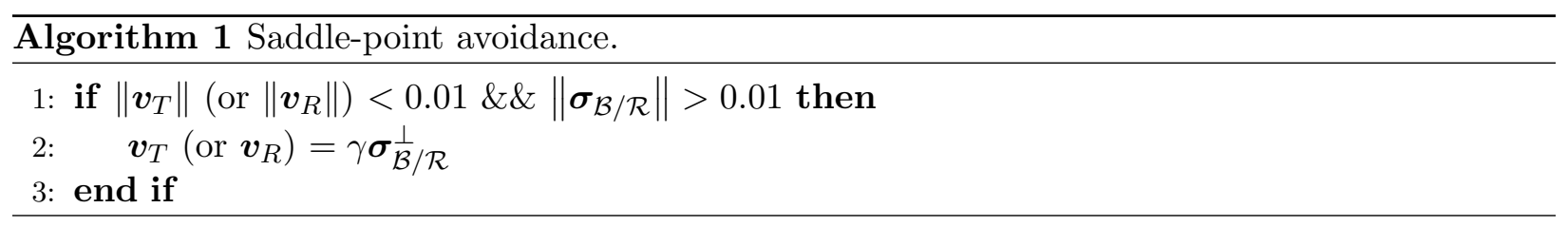




\subsection{Numerical Simulations}

Three different simulations are shown. Parameters are indicated in Table 4.1. Four identical reaction wheels in a pyramid configuration with an angle of $55^{\circ}$ are used. The four reaction wheels are spinning at nominal speed $(500 \mathrm{rpm})$.

The maximum permitted angular velocity is $\omega_{\max }=2^{\circ} / \mathrm{sec}$.

Four exclusion constraints are used. One inclusion constraint (not used in the regulation problems) is also setup. Different cameras and/or antennas (or solar panels) are defined in the examples.

The servo sub-system given in Equation (2.27) requires the computation of the body-frame derivative $\boldsymbol{\omega}_{\mathcal{B}^{*} / \mathcal{R}}^{\prime}$. This derivative is numerically computed ${ }^{6}$ using backward differences[33] and a 0.5-sec-window moving average 32 in order to smooth the signal and avoid unnecessary noise

Table 4.1: Simulation parameters.

\begin{tabular}{|c|c|c|}
\hline Description & Variables & Values \\
\hline Spacecraft & {$\left[I_{S}\right]\left[\mathrm{kg}-\mathrm{m}^{2}\right]$} & $\operatorname{diag}\left(\left[\begin{array}{lll}4.415 & 4.415 & 3.83\end{array}\right]\right)$ \\
\hline \multirow{5}{*}{ RW } & {$\left[I_{w}\right]\left[\mathrm{kg}-\mathrm{m}^{2}\right]$} & $\operatorname{diag}\left(\left[\begin{array}{lll}0.03 & 0.001 & 0.001\end{array}\right]\right)$ \\
\hline & & $\begin{array}{llll}0.819 & 0 & -0.819 & 0\end{array}$ \\
\hline & {$\left[G_{s}\right]$} & -0.819 \\
\hline & & $0.5736 \quad 0.5736$ \\
\hline & $u_{s_{\max }}$ & $15 \mathrm{mNm}$ \\
\hline \multirow{3}{*}{ Control Constants } & {$[P]$} & $10\left[I_{3 \times 3}\right]$ \\
\hline & {$\left[K_{I}\right]$} & $0.01\left[I_{3 \times 3}\right]$ \\
\hline & $K_{1}, K_{3}$ & 0.1 \\
\hline \multirow{4}{*}{ Exclusion Constraints } & $\mathcal{N}_{\hat{\boldsymbol{n}}_{1}}, \theta_{\min 1}$ & $-0.34 \quad-0.96]^{T}, 10^{\circ}$ \\
\hline & $\mathcal{N}_{\hat{\boldsymbol{n}}_{2}}, \theta_{\min 2}$ & {$\left[\begin{array}{lll}0 & -1 & 0\end{array}\right]^{T}, 30^{\circ}$} \\
\hline & $\mathcal{N}_{\hat{\boldsymbol{n}}_{3}, \theta_{\min 3}}$ & {$\left[\begin{array}{lll}1 & 1 & 0\end{array}\right]^{T}, 20^{\circ}$} \\
\hline & $\overline{\mathcal{N}}_{\hat{\boldsymbol{n}}_{4}, \theta_{\min 4}}$ & $-1 \quad 10^{T}, 20^{\circ}$ \\
\hline Inclusion Constraints & $\mathcal{N}_{\hat{\boldsymbol{n}}_{5}, \theta_{\min 5}}$ & {$\left[\begin{array}{lll}1 & 0 & 0\end{array}\right]^{T}, 70^{\circ}$} \\
\hline
\end{tabular}

\footnotetext{
${ }^{6}$ See Subsection 2.3 .3 for details on numerically computing the derivative.
} 


\subsubsection{Slew Problem}

In this first numerical simulation, a typical slew maneuver is shown. The spacecraft is taken from an arbitrary initial orientation to a final orientation aligned with the inertial frame. Two sensitive instruments are considered. The first, Camera 1, is in the $\boldsymbol{y}$-body direction, while the second, Camera 2, is in the $\boldsymbol{x}$-body direction. None of them must enter into the four exclusion zones given in Table 4.1. No inclusion zones are considered in this example. Since it is a typical slew maneuver, the initial angular velocity is zero.

Results are illustrated in Figure 4.2 Figure $4.2 \mathrm{~b}$ shows that the angular velocity is bounded ( $\omega_{1 d}, \omega_{2 d}, \omega_{3 d}$ indicate the steering law commands). The reaction wheels torques, in Figure $4.2 \mathrm{c}$, are also bounded 7 .

Since the problem does not have any symmetry, no saddle-points are reached, as is shown in Figure $4.2 \mathrm{~d}$. Indeed, the vector $\boldsymbol{v}_{R}$ is never zero except when $\boldsymbol{\sigma}_{\mathcal{B} / \mathcal{N}}=\mathbf{0}$.

The most interesting plots are shown in Figures $4.2 \mathrm{e}$ and $4.2 \mathrm{f}$, where a cylindrical projection of each camera's unit vector tip is plotted projected into a cylinder. The exclusion zones are the numerated blobs. The numbering corresponds to that given in Table 4.1. As can be seen, both cameras avoid the exclusion zones and reach their final destination. Camera 1, in the $\boldsymbol{y}$-body direction ends up aligned to the $\boldsymbol{y}$-inertial unit vector. This corresponds to a declination of $90^{\circ}$. Similarly, Camera 2, in the $\boldsymbol{x}$-body direction ends up aligned to the $\boldsymbol{x}$-inertial unit vector, that corresponds to a declination of $0^{8} 8$.

\subsubsection{Saddle-Points in the Regulation Problem}

In this example, the existence of a saddle-point is illustrated. In order to have a saddle-point, spatial symmetry relative to the initial conditions must exist. The symmetric condition is achieved with four exclusion and no inclusion constraints. Only Camera 1 is used.

\footnotetext{
7 The reason why the algorithm works with bounded control torque is discussed in Chapter 5 .

${ }^{8}$ It can be also thought in terms of latitude and longitude, where $\boldsymbol{x}$-inertial corresponds to $0^{\circ}$ latitude- $0^{\circ}$ longitude, and $\boldsymbol{y}$-inertial to $0^{\circ}$ latitude- $90^{\circ}$ longitude.
} 


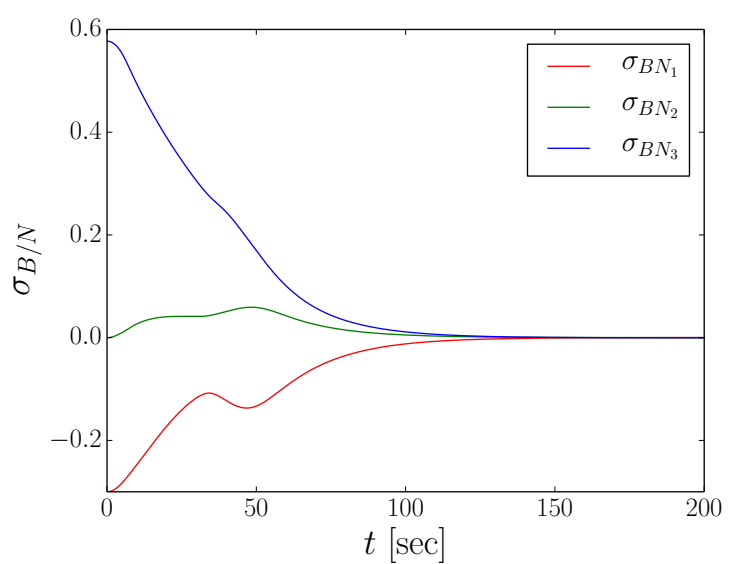

(a) Attitude $\boldsymbol{\sigma}_{\mathcal{B} / \mathcal{N}}$

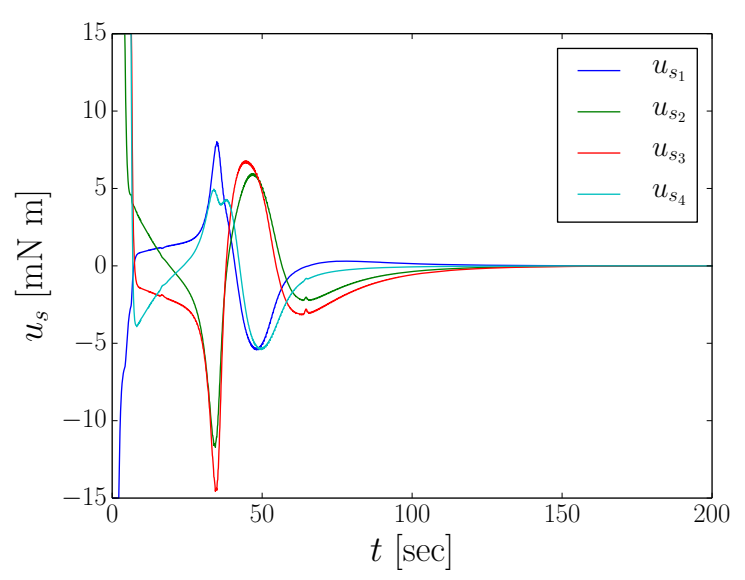

(c) Reaction Wheels' torques.

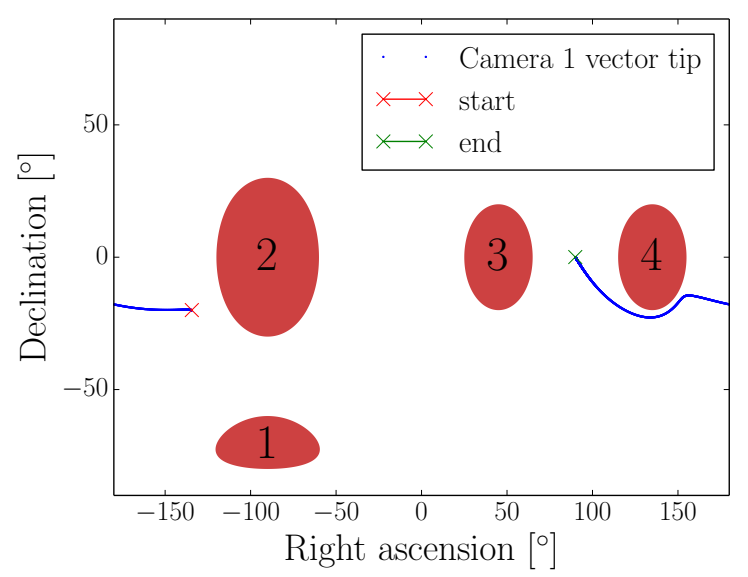

(e) Cylindrical projection of the Camera 1 unit (f) Cylindrical projection of the Camera 2 unit vector tip.

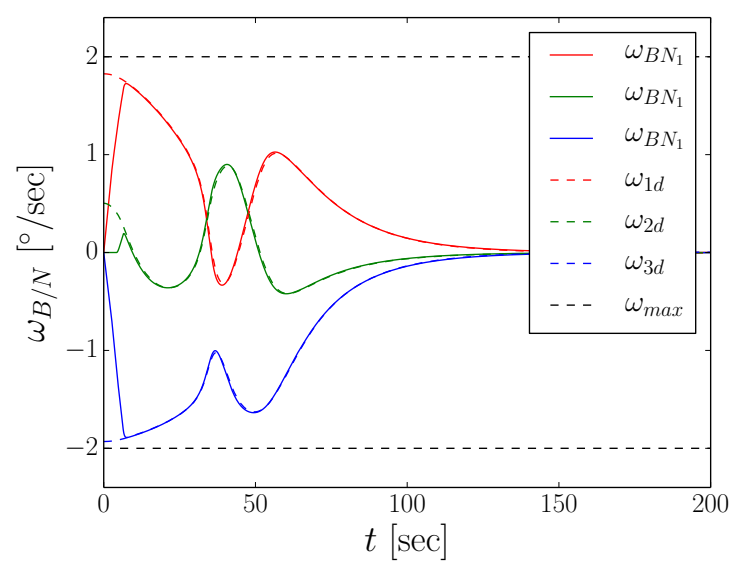

(b) Angular velocity $\boldsymbol{\omega}_{\mathcal{B} / \mathcal{N}}$.

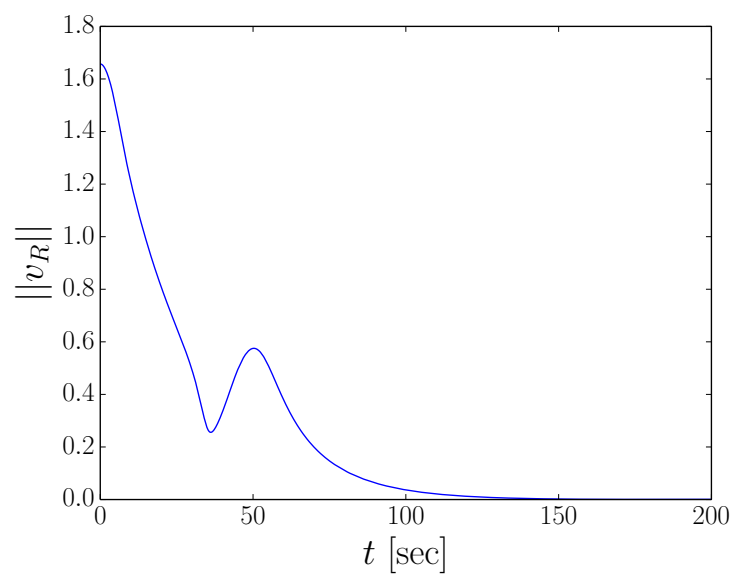

(d) Saddle-point indicator: $\left\|\boldsymbol{v}_{R}\right\|$.

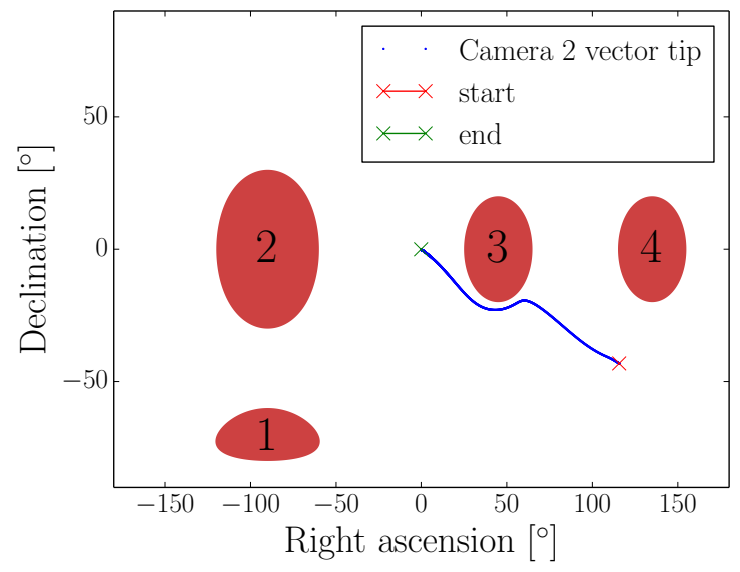
vector tip.

Figure 4.2: Regulation control performance illustration: Typical slew maneuver. 
The initial conditions are chosen as follows:

$$
\begin{aligned}
\boldsymbol{\sigma}_{\mathcal{B} / \mathcal{N}_{0}} & =\left[\begin{array}{lll}
-0.67 & 0 & 0
\end{array}\right]^{T} \\
\boldsymbol{\omega}_{\mathcal{B} / \mathcal{N}_{0}} & =\left[\begin{array}{lll}
\omega_{\max } & 0 & 0
\end{array}\right]^{T}
\end{aligned}
$$

The initial attitude is a simple rotation of $-135^{\circ}$ about the $\boldsymbol{x}$ axis. The initial angular velocity is full speed $\left(\omega_{\max }\right)$ in the same direction. In other words, Camera 1 vector's tip (in the $\boldsymbol{y}$-body axis) is rotating full speed into the first exclusion constraint. Given the four symmetric exclusion zones, the attitude initial condition is purposely picked to achieve a condition where a saddle-point may arise.

$$
\begin{aligned}
& \text { Algorithm }\left[1 \text { is used for saddle-point avoidance with } \gamma=0.01 \text { and } \boldsymbol{\sigma}_{\mathcal{B} / \mathcal{N}}^{\perp}=\left[\begin{array}{ccc}
-\sigma_{3} & 0 & \sigma_{1}
\end{array}\right]\right. \text { if } \\
& \sigma_{1} \neq \text { and } \sigma_{3} \neq 0 \text { and } \boldsymbol{\sigma}_{\mathcal{B} / \mathcal{N}}^{\perp}=\left[\begin{array}{lll}
\sigma_{2} & 0 & 0
\end{array}\right] \text { in other case. }
\end{aligned}
$$

Figure 4.3 shows the results. As can be seen in Figure $4.3 \mathrm{~b}$, the steering law generates a command of almost full speed in the opposite direction $\left(\omega_{1 d}, \omega_{2 d}, \omega_{3 d}\right.$ indicate the steering law commands) in order to brake the spacecraft. The situation is depicted in the cylindrical projection in Figure 4.3c. In this figure, the tip of the boresight vector in inertial space and the exclusion constraints (blobs) are projected into a right ascension-declination 2-D space. The dotted line indicates the path the unconstrained law would follow. The initial condition is such that the boresight vector is rotating straight into the near-south-pole constraint. The controller stops the rotation, reaches a saddle-point, and after using Algorithm 1 to getting out of it, rotates the spacecraft smoothly to the target.

For comparison, in Figure $4.3 \mathrm{~d}$ the fourth constraint is removed and the symmetry is broken. Thus, no saddle-point is reached and the control is smoother. The vector $\boldsymbol{v}_{R}$ is never zero except when $\boldsymbol{\sigma}_{\mathcal{B} / \mathcal{N}}=\mathbf{0}$.

Finally, Figure $4.3 \mathrm{e}$ shows the vector's norm $\left\|\boldsymbol{v}_{R}\right\|$ for the symmetric case depicted in Figure 4.3c. As can be seen, a saddle-point is reached when $\boldsymbol{v}_{R} \approx \mathbf{0}$. Figure 4.3f. on the other side, shows the vector's norm $\left\|\boldsymbol{v}_{R}\right\|$ for the asymmetric case depicted in Figure 4.3d, No saddle-point is ever reached in this case. 


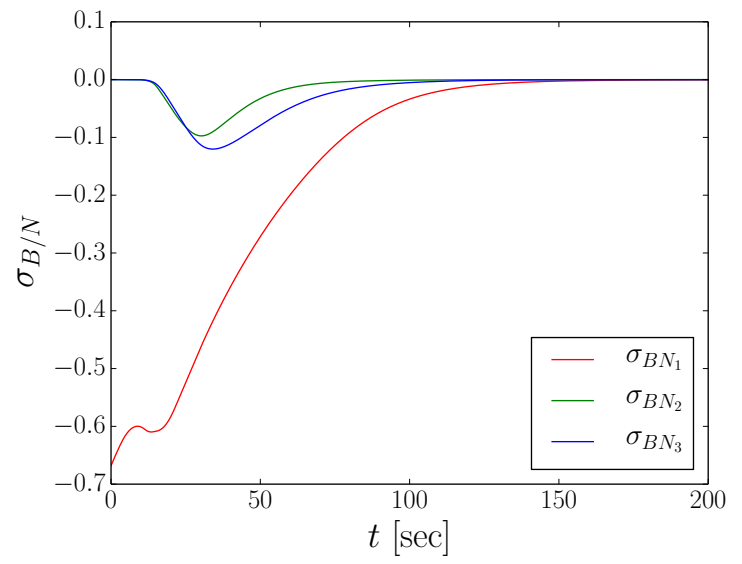

(a) Attitude $\sigma_{\mathcal{B} / \mathcal{N}}$.

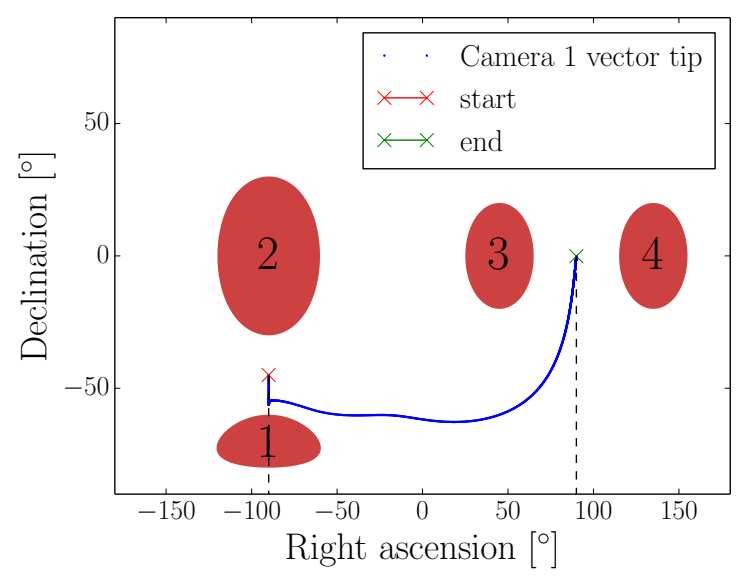

(c) Cylindrical projection of the Camera 1 unit vector tip with symmetric conditions.

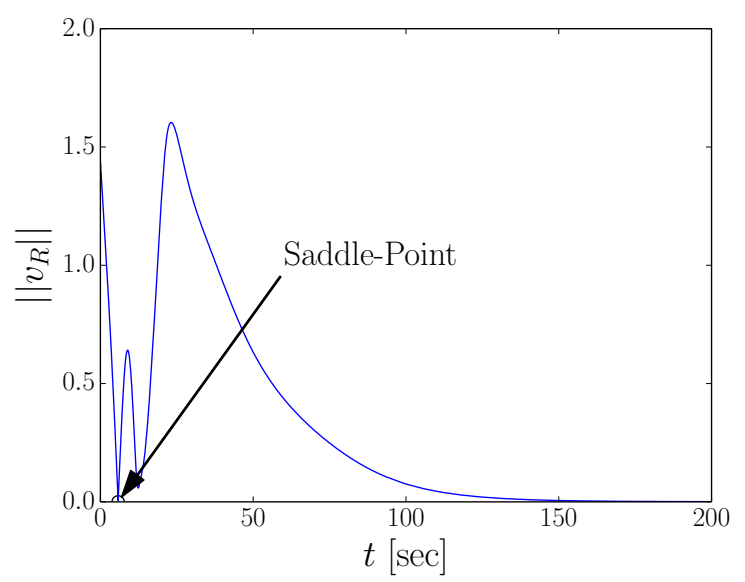

(e) Saddle-point indicator: $\left\|\boldsymbol{v}_{R}\right\|$ with symmetric (f) Saddle-point indicator: $\left\|\boldsymbol{v}_{R}\right\|$ with asymmetconditions.

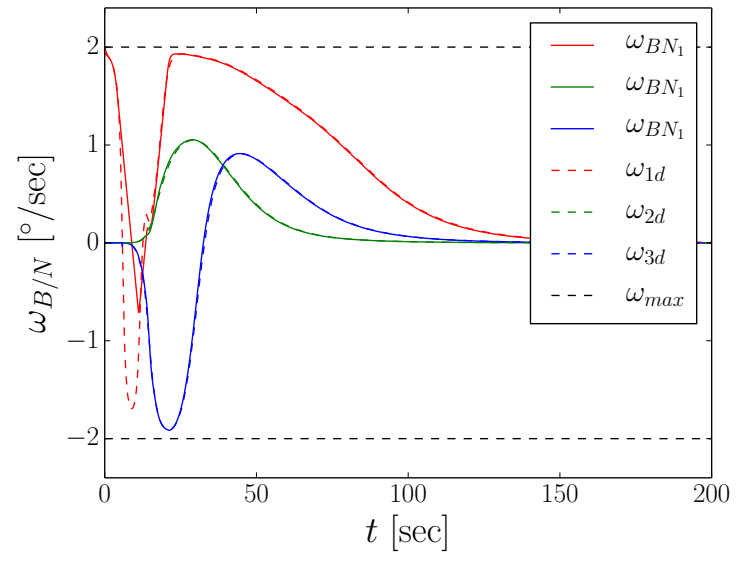

(b) Angular velocity $\boldsymbol{\omega}_{\mathcal{B} / \mathcal{N}}$.

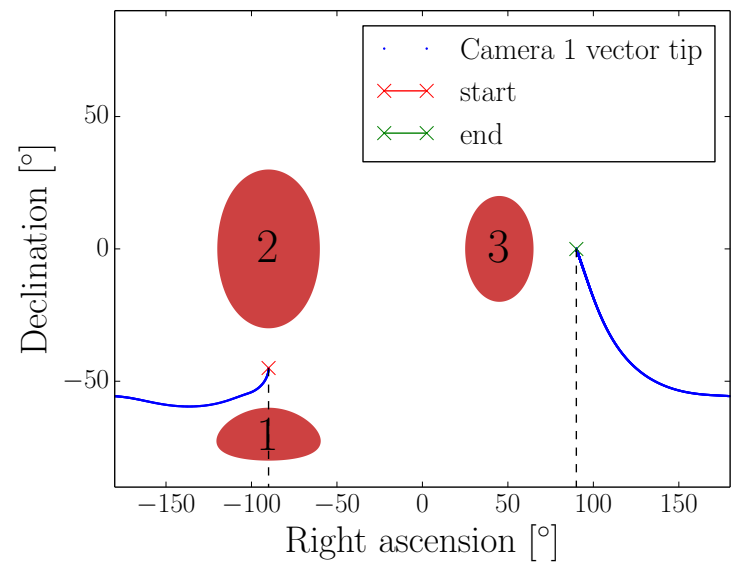

(d) Cylindrical projection of the Camera 1 unit vector tip with asymmetric conditions.

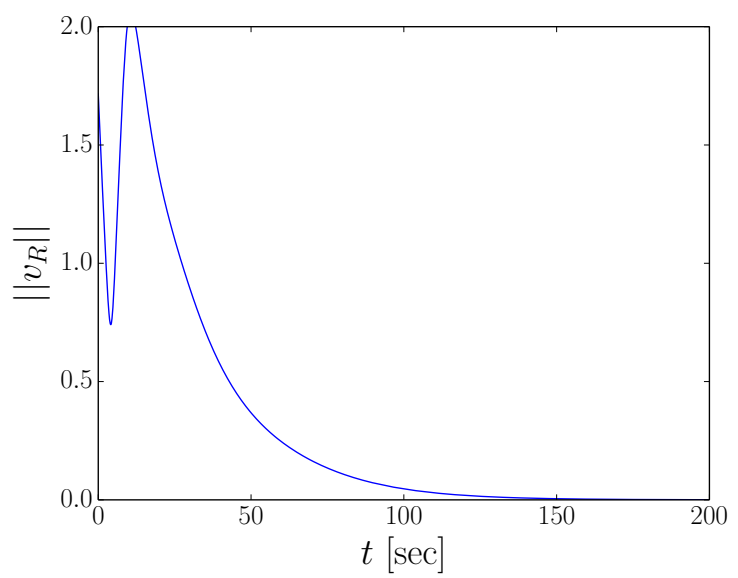

ric conditions.

Figure 4.3: Regulation control performance illustration: Saddle-point avoidance. 


\subsubsection{Tracking Problem}

This simulation shows a tracking example using the four exclusion and one inclusion constraints given in Table 4.1. One sensitive instrument is considered. Camera 1 is in the $\boldsymbol{y}$-body direction. An antenna in the $\boldsymbol{x}$-body direction has to point somewhere inside the inclusion constraint in Table 4.1.

The reference frame $\mathcal{R}$ is constructed as follows. In a circular orbit around a perfectly spherical Earth, the direction $\hat{\boldsymbol{r}}_{1}$ is the nadir direction. $\hat{\boldsymbol{r}}_{3}$ is the angular momentum direction, normal to the orbit, and $\hat{\boldsymbol{r}}_{2}=\hat{\boldsymbol{r}}_{3} \times \hat{\boldsymbol{r}}_{1}$. Thus, $[R N]=\left[\begin{array}{lll}\mathcal{N}_{\hat{\boldsymbol{r}}_{1}} & \mathcal{N}_{\hat{\boldsymbol{r}}_{2}} & \mathcal{N}_{\hat{\boldsymbol{r}}_{3}}\end{array}\right]^{T}$. The orbital parameters are shown in Table 4.2 .

Table 4.2: Reference frame parameters.

\begin{tabular}{c|c}
\hline \hline Description & Values \\
\hline \hline Earth radius & $6378.0 \mathrm{~km}$ \\
\hline Earth's gravitational parameter & $398600.0 \mathrm{~km} / \mathrm{sec}^{2}$ \\
\hline Right ascension of ascending node & $0^{\circ}$ \\
\hline Inclination & $-90^{\circ}$ \\
\hline Orbit altitude & $400 \mathrm{~km}$ \\
\hline Initial argument of latitude & $180^{\circ}$ \\
\hline \hline
\end{tabular}

The results can be seen in Figure 4.4. The cylindrical projections of the exclusion and inclusion zones in Figures $4.4 \mathrm{e}$ and $4.4 \mathrm{f}$ show that the reference is tracked without violating any constraint. This can be further corroborated in Figures $4.4 \mathrm{c}$ and $4.4 \mathrm{~d}$, which illustrate attitude and rate errors. 


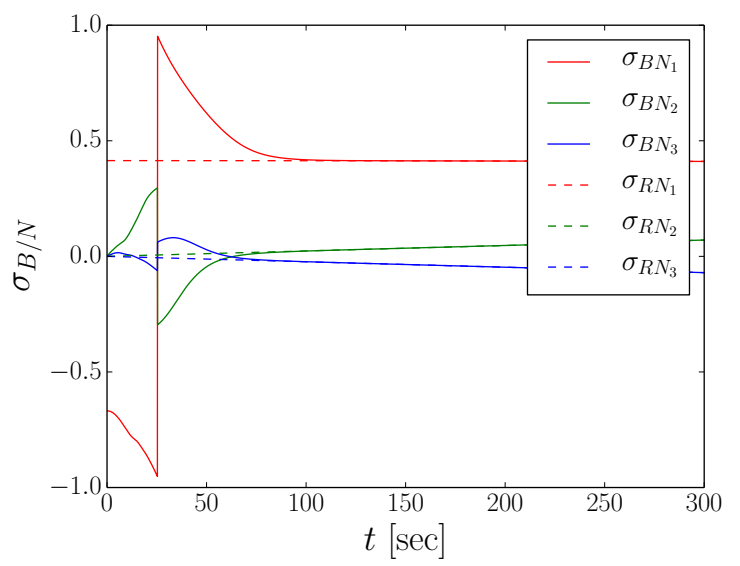

(a) Attitude $\sigma_{\mathcal{B} / \mathcal{N}}$.

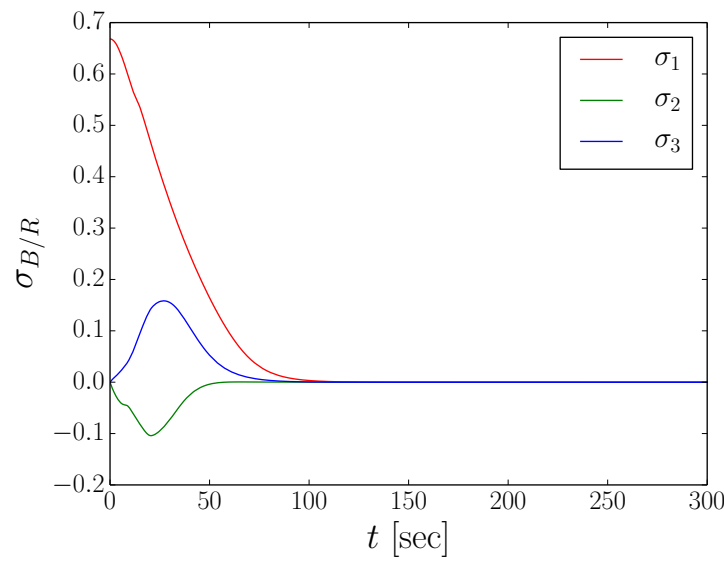

(c) Attitude error $\boldsymbol{\sigma}_{\mathcal{B} / \mathcal{N}}$.

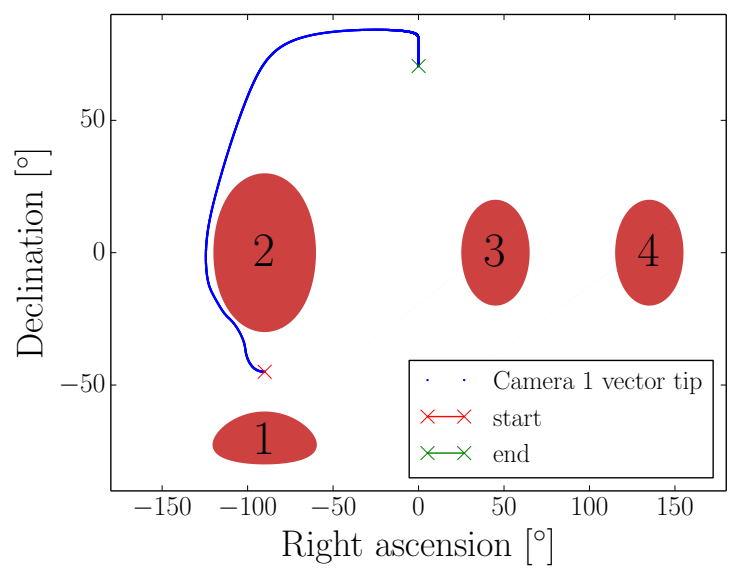

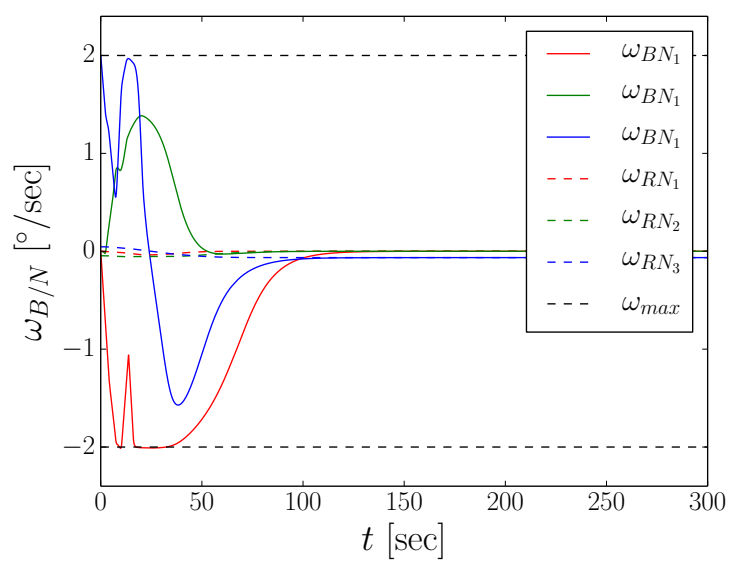

(b) Angular velocity $\boldsymbol{\omega}_{\mathcal{B} / \mathcal{N}}$.

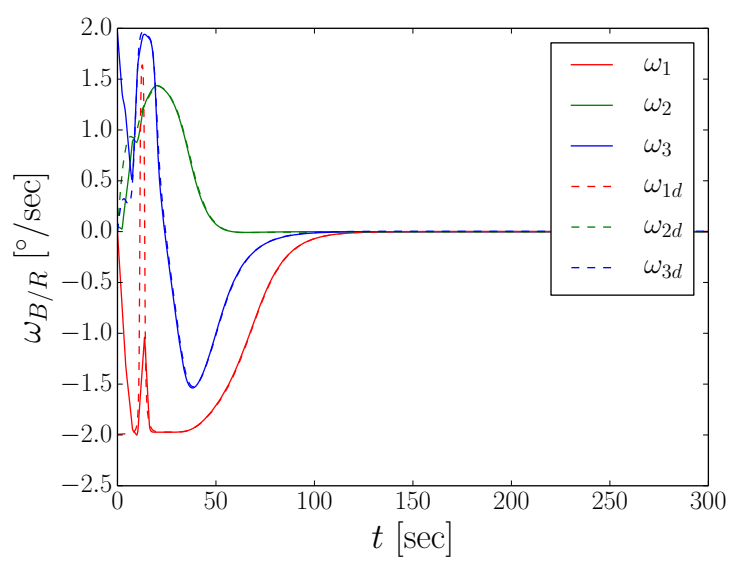

(d) Angular velocity error $\boldsymbol{\omega}_{\mathcal{B} / \mathcal{R}}$.

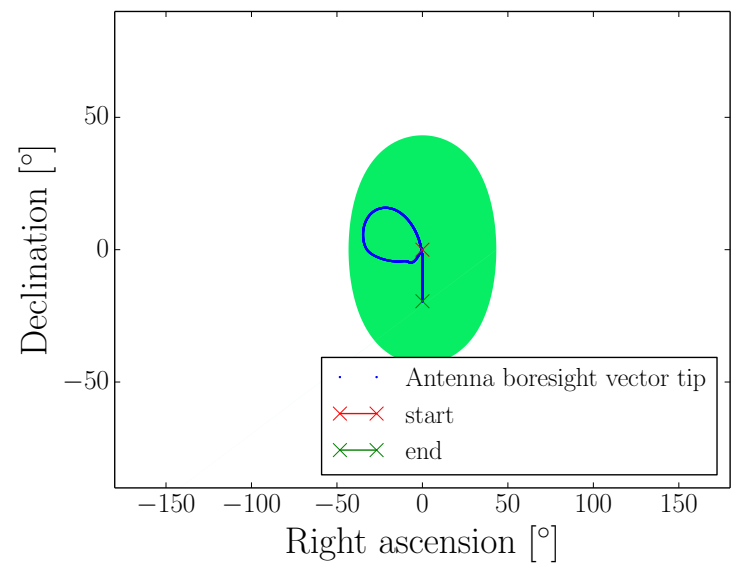

(e) Cylindrical projection of the Camera 1 unit (f) Cylindrical projection of the antenna unit vector tip. vector tip.

Figure 4.4: Tracking control performance illustration. 


\section{Chapter 5}

\section{The Effect of Bounded Control Torque}

In this Chapter, the effect of a bounded control torque on the attitude constraint law is studied.

\subsection{Constraint avoidance with finite control torque}

Even though the regulation control law given in Equation (4.10) is bounded in angular velocity, it might require infinite angular acceleration when close to a constraint. In order to study how the control law performs with limited torque capacity, Figure 5.1 considers a very simple planar scenario. The boresight vector is rotating on a plane straight into a constraint cone. The inertia of the system about the fixed rotation axis is $I_{\max }$, the angular velocity is $\omega$ and a constant available torque is given by $u_{\max }$. The angle at time $t_{0}$ is $\theta_{0}$ and the initial angular velocity is $\omega_{0}$. The problem can be stated as follows: with inertia $I_{\max }$, constant control torque $u_{\max }$, and initial velocity $\omega_{0}=\omega_{\max }$ given, what is the initial angle $\theta_{0}$ to exactly stop the rotation at the security cone given by the angle $\theta_{\min }$ ?

Since $\omega=-\dot{\theta}$, it is possible to write

$$
\begin{aligned}
\ddot{\theta} & =-\dot{\omega}=\frac{u_{\max }}{I_{\max }} \\
\dot{\theta}_{f} & =\dot{\theta}\left(t_{f}\right)=0=\frac{u_{\max }}{I_{\max }}\left(t_{f}-t_{0}\right)-\omega_{\max } \\
\theta_{f} & =\theta\left(t_{f}\right)=\theta_{\min }=\frac{1}{2} \frac{u_{\max }}{I_{\max }}\left(t_{f}-t_{0}\right)^{2}-\omega_{\max }\left(t_{f}-t_{0}\right)+\theta_{0}
\end{aligned}
$$




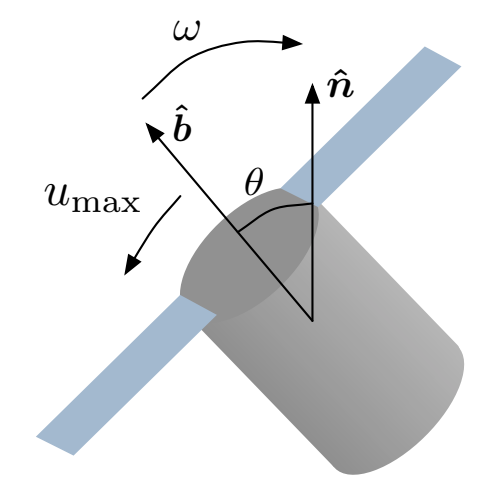

Figure 5.1: Worst-case scenario. The spacecraft is rotating in a fixed plane at maximum angular velocity straight into a constraint. $u_{\max }$ is the maximum torque capacity in that direction.

Solving for $\left(t_{f}-t_{0}\right)$ in the first equation and replacing into the second

$$
\theta_{0}=\frac{1}{2} \frac{I_{\max }}{u_{\max }} \omega_{\max }^{2}+\theta_{\min }
$$

For a given spacecraft, $I_{\max }$ should be the maximum axis of inertia, $\omega_{\max }$ is the maximum velocity used in the steering law in Equation 2.16) and $u_{\max }$ should be, at most, the maximum torque available in the poorest controllable direction.

Given a maximum torque for each wheel $u_{s_{\max }}$, the minimum torque capacity for a RW array can be computed using torque envelopes. The algorithm for computing this minimum torque capacity is given in Reference [21]. An $N_{\mathrm{RW}}$-wheel system has an $N_{\mathrm{RW}}$-dimensional torque vector $\boldsymbol{u}_{\mathrm{S}}$ feasible set that fills the interior of a hypercube of dimension $N_{\mathrm{RW}}$, where each side has a length of twice the maximum torque $\left(2 u_{s_{\max }}\right)$. This hypercube is mapped into a polyhedron in $3-\mathrm{D}$ space using the projection matrix $\left[G_{\mathrm{s}}\right]$ presented in Chapter 2. The mapping preserves vertices (all wheels saturated), edges (all but one wheel saturated), and facets (all but two wheels saturated), even though it is not an isomorphism. Moreover, some vertices, edges, and facets of the hypercube are mapped to the interior of the polyhedron. The algorithm given by Landis Markley et. al. [21] calculates the minimum torque ${ }^{1}$ of each outer facet to compute the minimum torque capacity as the minimum over all the facets. The maximum control torque magnitude that can be exerted by

\footnotetext{
${ }^{1}$ The paper describes the calculation of the minimum angular momentum, but they are both equivalent through a linear isomorphism.
} 
the RW array in the poorest controllable direction $\left(u_{\text {cap }}\right)$ can be computed as follows

$$
\begin{aligned}
u_{\text {cap }} & =\min \left\{u_{i, j \min } ; i, j=1, \ldots, N_{\mathrm{RW}}\right\} \\
u_{i, j \min } & =u_{s_{\max }} \sum_{k=1, k \neq i, j}^{N_{\mathrm{RW}}}\left|\hat{\boldsymbol{g}}_{s_{k}} \cdot \hat{\boldsymbol{n}}_{i j}\right| \\
\hat{\boldsymbol{n}}_{i j} & =\frac{\hat{\boldsymbol{g}}_{s_{i}} \times \hat{\boldsymbol{g}}_{s_{j}}}{\left\|\hat{\boldsymbol{g}}_{s_{i}} \times \hat{\boldsymbol{g}}_{s_{j}}\right\|}
\end{aligned}
$$

The $u_{\text {max }}$ used in Equation 5.4 can be a fraction of the torque capacity in Equation 5.5 .

$$
u_{\max }=\alpha u_{\text {cap }}, \quad 0<\alpha \leq 1
$$

Thus, for each exclusion constraint there is an angle $\theta_{\min }$ that defines the exclusion cone (inner cone) and an angle $\theta_{0}$ that defines an outer cone. Using $\theta_{\min }$ in the constraint functions in Eqs. 4.10) and (4.20), the finite-torque control will not break through any exclusion constraint as long as the initial attitude is outside any outer cone and the angular velocity is not greater than $\omega_{\max }$.

\subsection{Switching between the constrained and unconstrained laws}

An additional use of the result obtained in the last section enables switching between the constrained and unconstrained laws. When "far" from an exclusion constraint, it is possible to dismiss its inclusion in the control laws given by Eqs. (4.10) and (4.20). The angle $\theta_{0}$ in Equation (5.4) is used to evaluate this condition. The algorithm is shown as Algorithm 2, In order to avoid chattering, two different thresholds are defined using a hysteresis or Schmitt trigger [39] approach with a gap $\psi$. This algorithm is repeated for every single exclusion constraint. Therefore, at a given instant of time, some constraints are considered while others are not. That means eliminating those constraints that are not being utilized in Eqs. (4.8), (4.17) and (4.18).

In order to reduce (but not eliminate) the discontinuity while switching, the parameter $\alpha_{i}$ in Equation 4.18 can be chosen as

$$
\alpha_{i}=\left|\cos \left(\frac{1}{2} \frac{I_{\max }}{u_{\max }} \omega_{\max }^{2}+\theta_{\min i}\right)-\cos \left(\theta_{\min i}\right)\right| e
$$




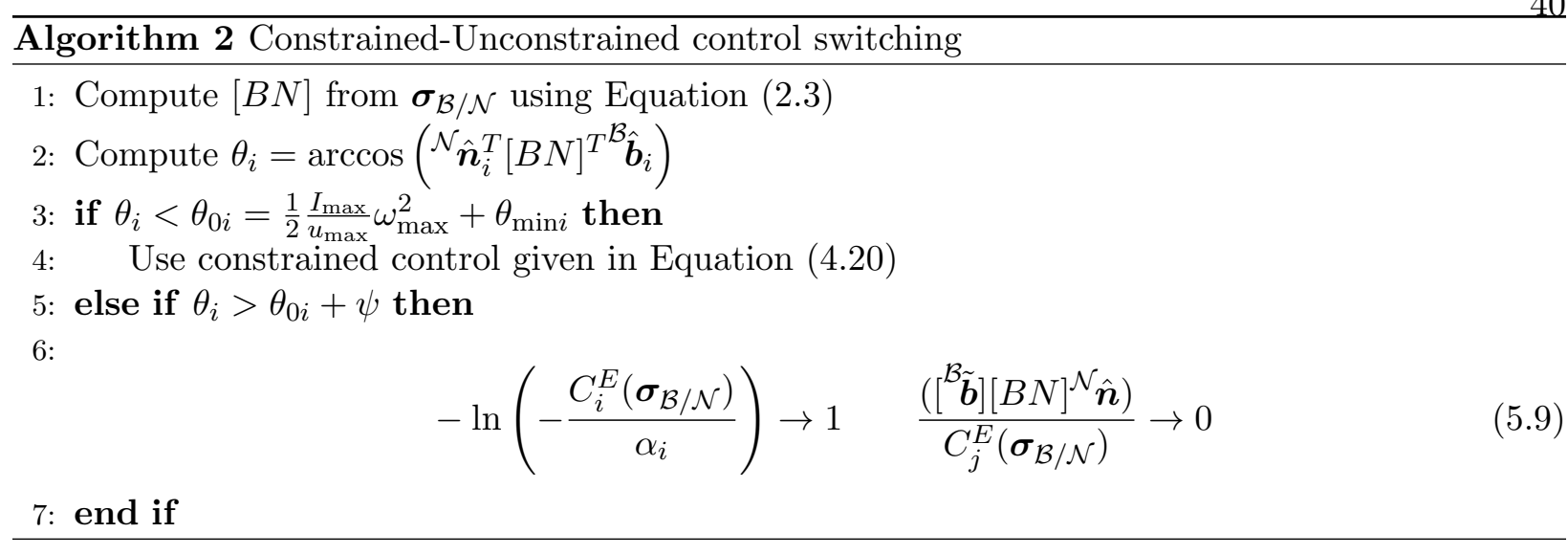

such that the logarithm in Equation (5.9) switches continuously when turning off the constraint algorithm.

\subsection{Numerical Simulations}

In this section, two different simulations are shown with the parameters indicated in Table 4.1 that have already been used in Chapter 4.

\subsubsection{Monte Carlo simulation}

A 50-run Monte Carlo simulation is shown in Figure 5.2. A regulation problem using exclusion constraints 1 and 2 in Table 4.1 is simulated 50 times for different initial conditions. The goal of the simulation is to statistically test the condition given by Equation (5.4). To that end, different initial attitudes are generated such that the boresight vector of a camera is always rotating straight into the first exclusion constraint at maximum angular velocity.

The initial attitude is random. The boresight vector of a camera is also randomly picked in some point on the outer cone of the first exclusion zone, computed using Equation (5.4). The initial angular velocity is then calculated such that the boresight vector is rotating straight into the constraint with magnitude $\omega_{\max }$. With this algorithm, different torque facets of the torque envelope are tested ${ }^{2}$.

\footnotetext{
${ }^{2}$ See Section 5.1 .
} 
In the cylindrical projection in Figure $5.2 \mathrm{~d}$, every single trajectory starts at the outer cone of the first exclusion constrained defined by the angle $\theta_{0}$ in Equation (5.4). The outer cone in Figure $5.2 \mathrm{~d}$ is represented by a lighter blob around the exclusion zone numbered as 1 . This is shown in Figure 5.2c, where the exclusion constraint angle $\theta_{1}=\arccos \left(\hat{\boldsymbol{n}}_{1} \cdot \hat{\boldsymbol{b}}_{1}\right)$ at the start of every run corresponds to $\theta_{0}$. As can be seen, the constraints are avoided with bounded angular velocity and limited torque (the maximum torque of each RW is $15 \mathrm{mNm}$, see Figure $5.2 \mathrm{~b}$ ), as long as the initial attitude is outside the outer cone. In Figure $5.2 \mathrm{c}$, the angle $\theta_{1}$ is never below $\theta_{\min }$. This is a key result, since it shows that it is possible to avoid piercing an exclusion conic constraint, even with limited torque, if the initial condition is outside an outer cone. The minimum angle defining this outer cone is related to the torque capacity of the RW configuration. 


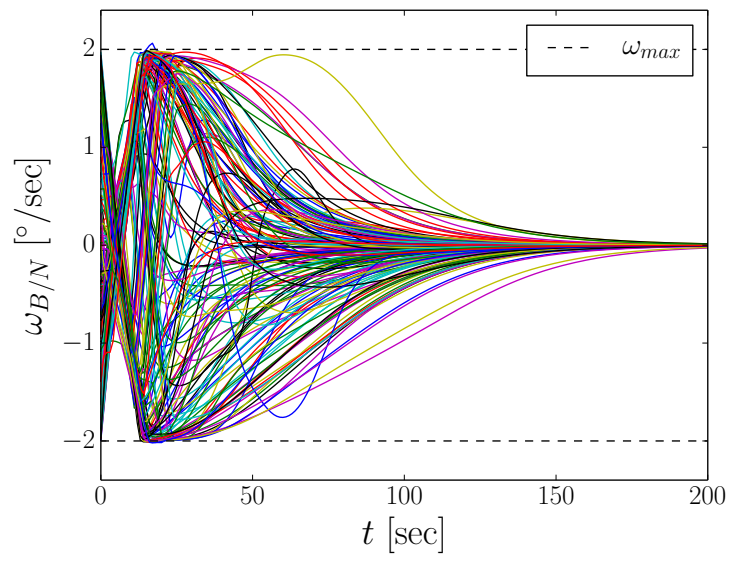

(a) Angular velocity $\boldsymbol{\omega}_{\mathcal{B} / \mathcal{N}}$.

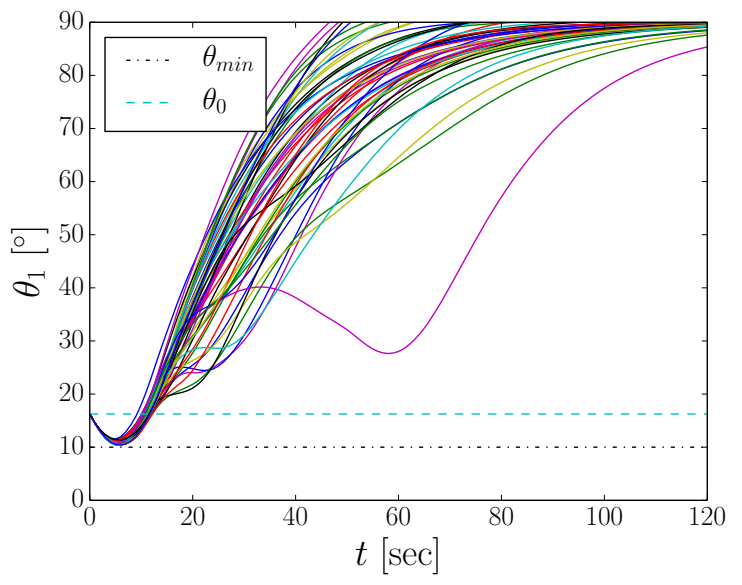

(c) Angle between $\hat{\boldsymbol{n}}_{1}$ and $\hat{\boldsymbol{b}}_{1}$.

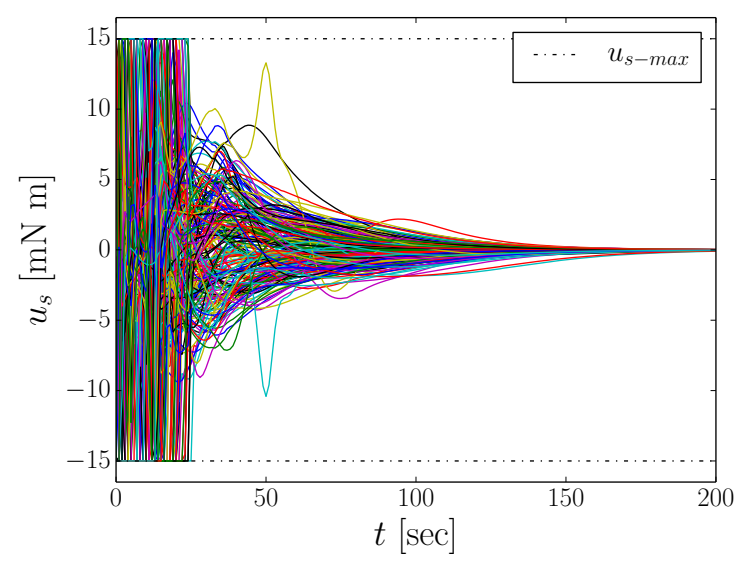

(b) RW torque.

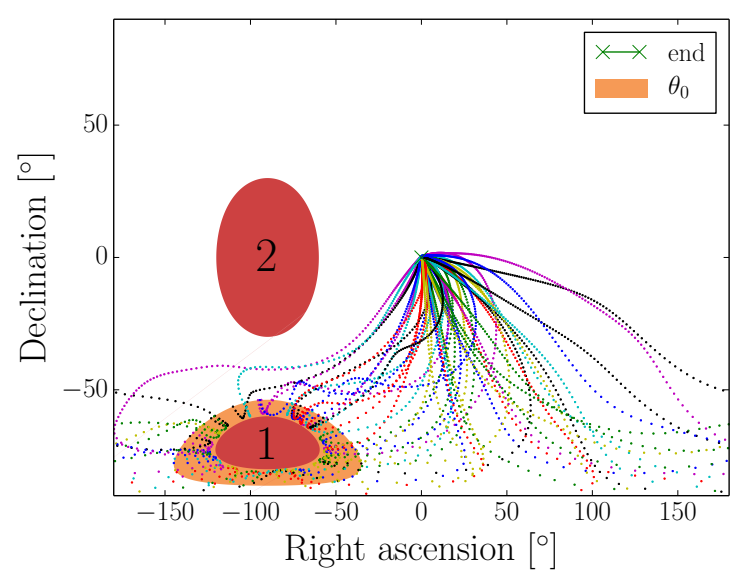

(d) Cylindrical projection map.

Figure 5.2: Monte Carlo simulation. 


\subsubsection{Tracking Problem With Constrained-Unconstrained Switching}

The simulation given in Subsection 4.5 .3 is repeated with the inclusion of Algorithm 2 for switching between the constrained and unconstrained laws using a gap of $\psi=5^{\circ}$. In Equation (5.4), $u_{\max }$ is chosen to be $50 \%$ of the minimum torque capacity computed with Equation (5.5).

The results can be seen in Figure 5.3. The cylindrical projections of the exclusion and inclusion zones in Figs. 5.3e and 5.3f show that the reference is tracked without violating any constraint.

The effect of using Algorithm 2 is shown in Figure 5.4, where the four exclusion constraint

angles $\theta_{1}, \theta_{2}, \theta_{3}, \theta_{4}$ and the constraint minimum $\left(\theta_{\min }\right)$ and threshold $\left(\theta_{0}\right)$ angles are plotted. The third and fourth exclusion constraints in Table 4.1 are so far from the trajectory that are not even considered by the control algorithm. The algorithm switches on and off constraints 1 and 2 using the threshold given in Equation (5.4) and the hysteresis gap given by $\psi=5^{\circ}$. After approximately 50 seconds, no constraint is used anymore and the purely unconstrained law is utilized instead.

It is interesting to compare Figure 5.3 with Figure 4.4 , obtained in Subsection 4.5.3. When switching constraints off, a discontinuity occurs, due to the following change in Algorithm 2

$$
\frac{\left.\left({ }^{\mathcal{B}} \tilde{\boldsymbol{b}}\right][B N]^{\mathcal{N}} \hat{\boldsymbol{n}}\right)}{C_{j}^{E}\left(\boldsymbol{\sigma}_{\mathcal{B} / \mathcal{N}}\right)} \rightarrow 0
$$

This discontinuity, which can be eliminated using filtering, can be seen as a sudden change in the angular velocity (compare Figures $5.3 \mathrm{~b}$ and $4.4 \mathrm{~b}$ ). 


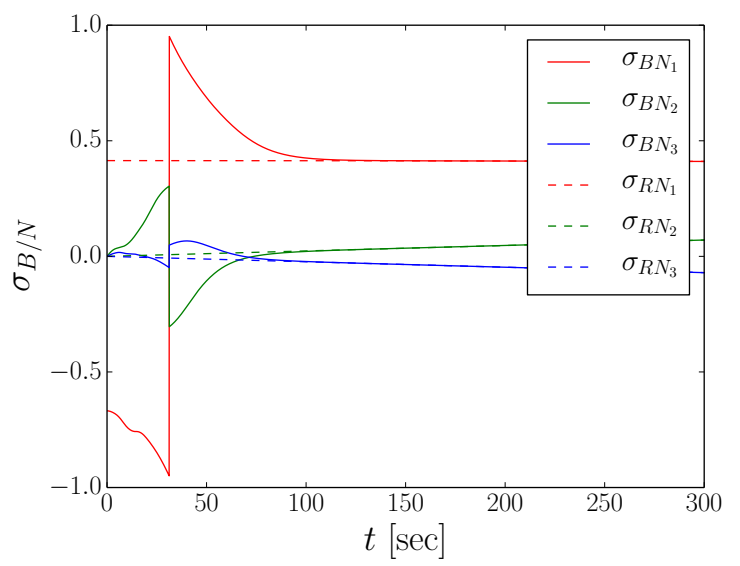

(a) Attitude $\sigma_{\mathcal{B} / \mathcal{N}}$.

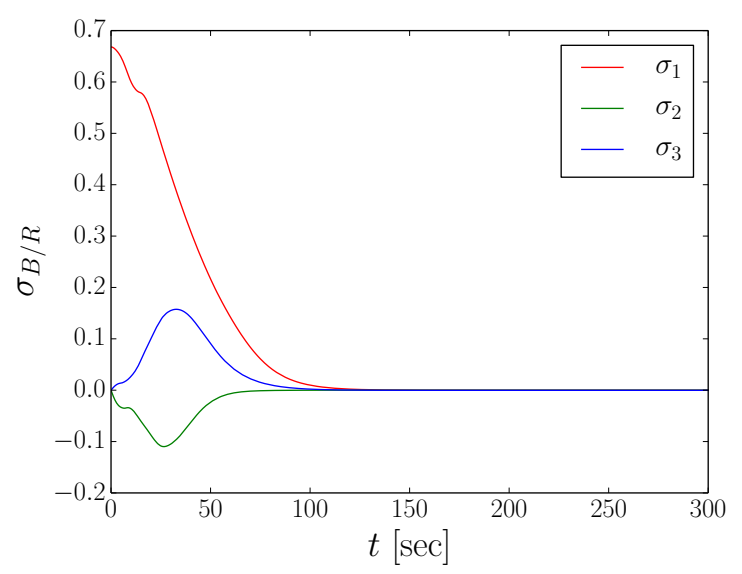

(c) Attitude error $\boldsymbol{\sigma}_{\mathcal{B} / \mathcal{N}}$.

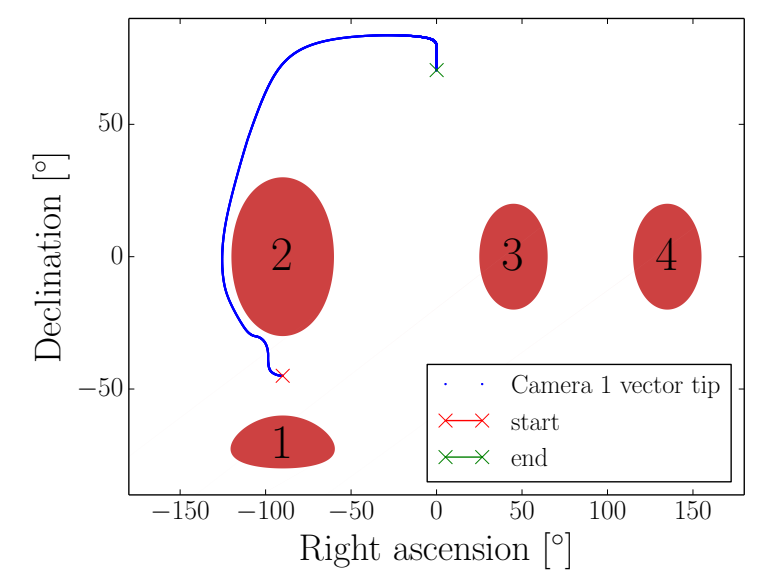

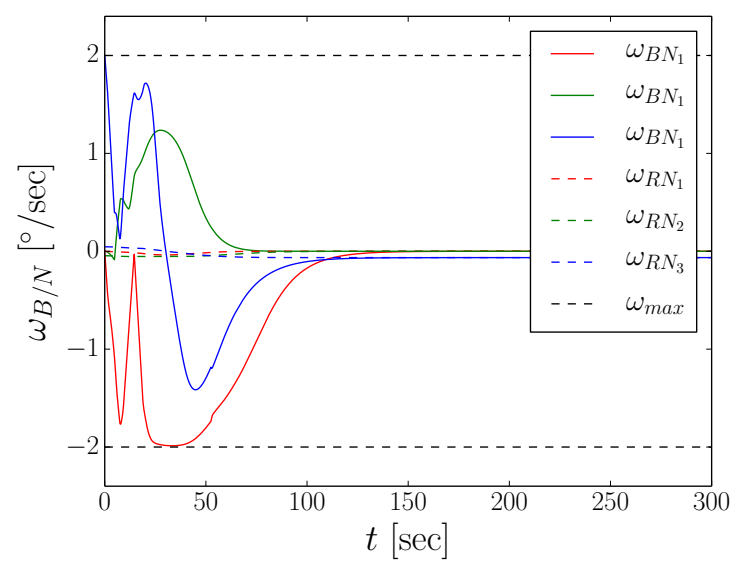

(b) Angular velocity $\boldsymbol{\omega}_{\mathcal{B} / \mathcal{N}}$.

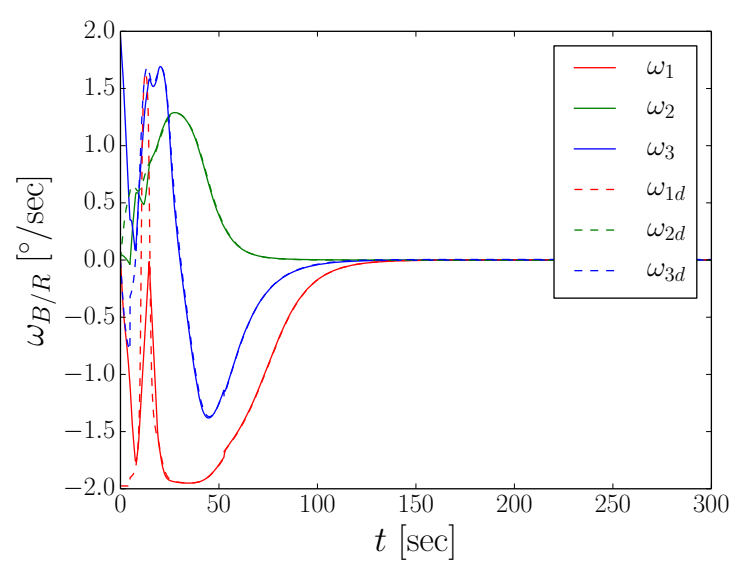

(d) Angular velocity error $\boldsymbol{\omega}_{\mathcal{B} / \mathcal{R}}$.

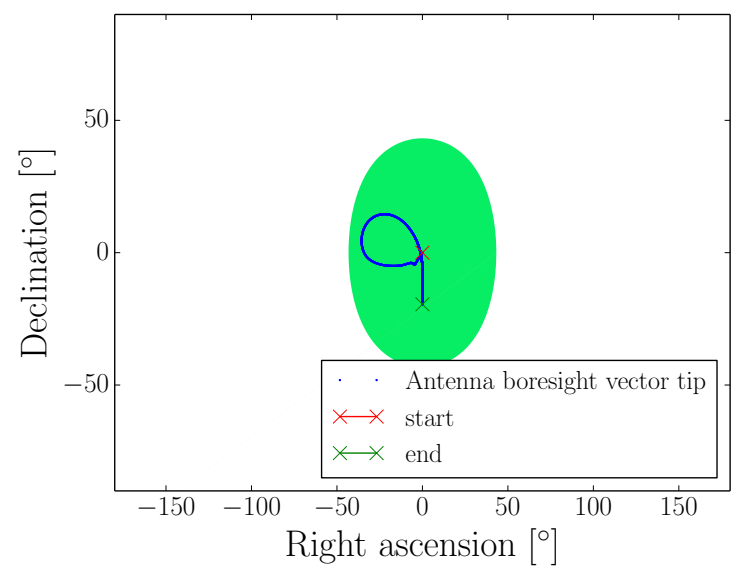

(e) Cylindrical projection of the Camera 1 unit (f) Cylindrical projection of the antenna unit vector tip. vector tip.

Figure 5.3: Tracking control performance illustration using contrained-unconstrained switching. 


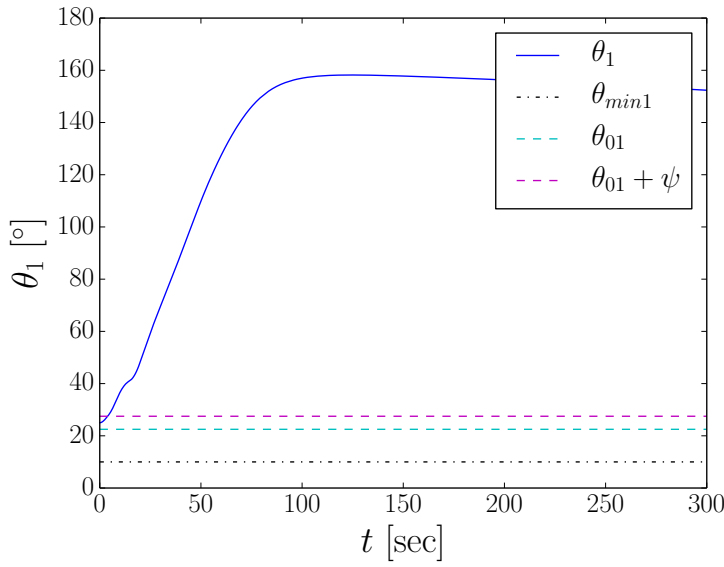

(a) Angle between $\hat{\boldsymbol{b}}_{1}$ and $\hat{\boldsymbol{n}}_{1}$.

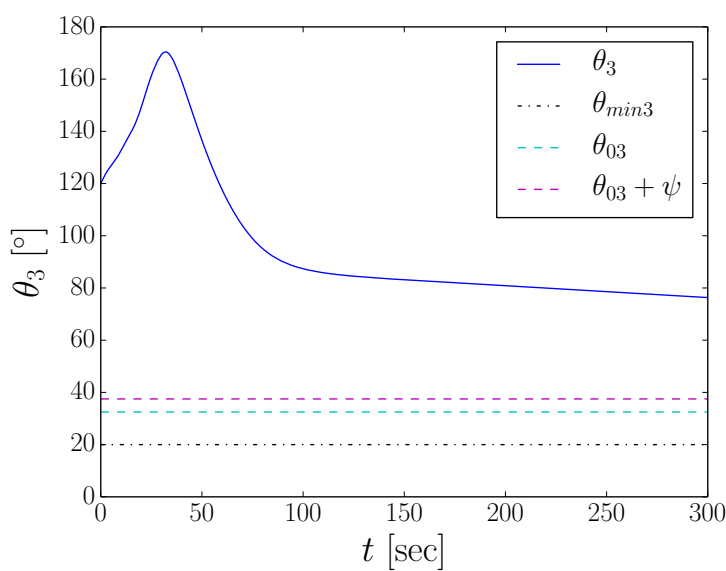

(c) Angle between $\hat{\boldsymbol{b}}_{3}$ and $\hat{\boldsymbol{n}}_{3}$.

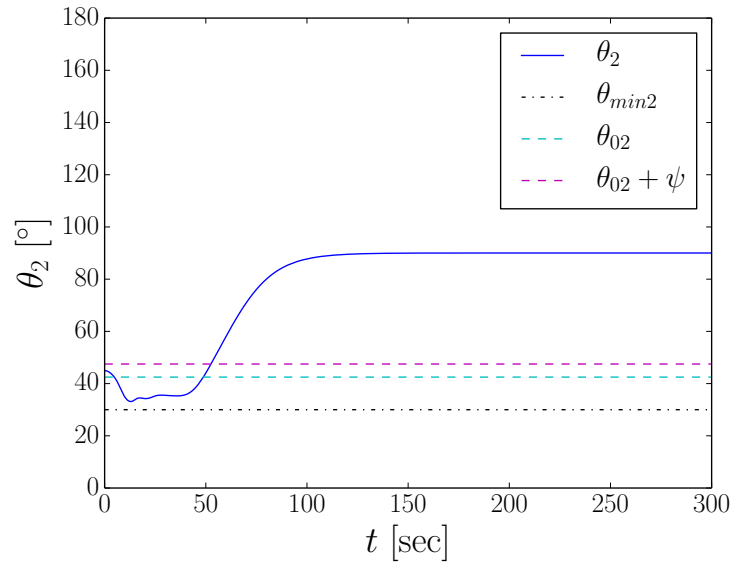

(b) Angle between $\hat{\boldsymbol{b}}_{2}$ and $\hat{\boldsymbol{n}}_{2}$.

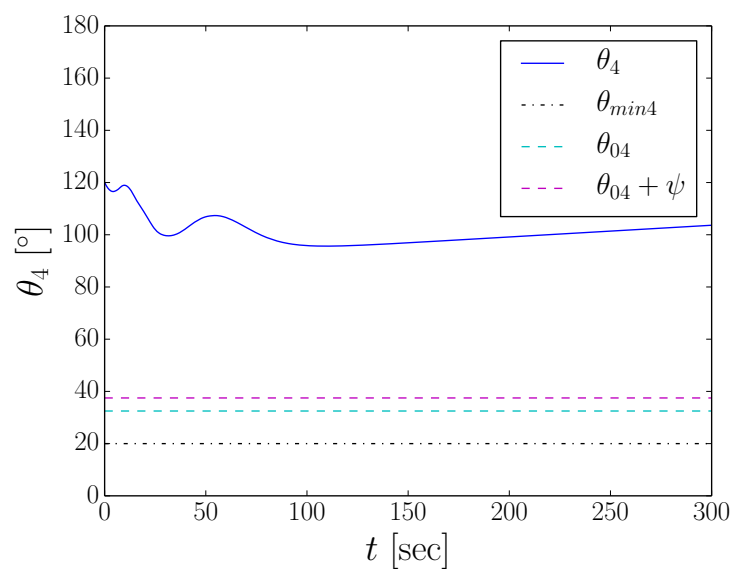

(d) Angle between $\hat{\boldsymbol{b}}_{4}$ and $\hat{\boldsymbol{n}}_{4}$.

Figure 5.4: Tracking control performance illustration using contrained-unconstrained switching. Exclusion constraint angles. Exclusion zones 3 and 4 are not considered by Algorithm 2 because they are "far" from the trajectory. 


\section{Chapter 6}

\section{Conclusions}

Orientation-constrained attitude control is not a mature technology. In fact, it is a lively active research topic with several different proposed solutions. Existing frameworks have different advantages and disadvantages. CMT has an inherent simplicity and has been successfully used in two missions. However, it might not be generalized because convergence cannot be demonstrated. Optimization techniques can take into account several different types of constraints, including bounded rates and torques, but yield an open-loop solution. Additionally, optimal methods are algorithmically complex and cannot be applied to tracking without modification. Graph methods are closed-loop, but do not solve the tracking problem and also have high complexity.

On the other hand, Lyapunov-derived methods have low complexity from an algorithmic point of view. Unfortunately, the current available techniques do not limit angular rate and control torque, which makes them hardly applicable to real attitude constrained control problems. Furthermore, they do not solve the tracking problem either.

One of the main advantages of using Lyapunov theory is, in fact, to be able to synthesize control laws that, though nonlinear, are fairly simple and ideal to real-time attitude control. Indeed, few closed-form function evaluations are usually required. This thesis extends the benefits of using Lyapunov-derived methods to rate-and-torque-bounded problems. Furthermore, the tracking problem with orientation constraints is also solved.

Bounded angular velocity is achieved in the regulation problem using a kinematic steering law to control the attitude of a spacecraft with reaction wheels under static hard conic constraints. 
The algorithm works well with any number of constraints, even in highly-symmetric conditions.

Bounded control torque can be achieved by extending the exclusion cones to take into account the limited control torque capacity of the spacecraft. The extended cones are not much larger than the original exclusion cones, being a function of the minimum torque capability of the reaction wheel array of the spacecraft. As a corollary, this result makes it possible, if desired, to switch from the constrained steering law to the unconstrained steering law when sufficiently far from the constraint cones.

As an additional characteristic, the control algorithms described use Modified Rodrigues Parameters as attitude descriptors. The use of MRPs in this problem provides a minimal attitude parameterization that yields globally non-singular behavior with short-rotation descriptions.

There are several questions that can be addressed in future work. First, the algorithm for escaping from saddle points has been explained heuristically, but not mathematically. Second, the algorithm switching between constrained and unconstrained laws still present some discontinuities. Future work could address further smoothing techniques for switching between both. Third, the tracking law given in Eq. 4.20 is not necessarily bounded in angular velocity because it depends on the nature of the reference. However, it has been seen in the simulations that the angular velocity remains bounded in spite of the second term in Eq. 4.20). This condition needs further study and could be addressed in the future. Fourth, this work deals only with static hard conic constraints. Extending the results to dynamic hard conic constraints, where the axis of the cone is not inertially fixed, might be possible. 


\section{Bibliography}

[1] A. Ahmed, J. Alexander, D. Boussalis, W. Breckenridge, G. Macala, M. Mesbahi, M. San Martin, G. Singh, and E. Wong. Cassini Control Analysis Book. Jet Propulsion Laboratory, 1998.

[2] S. Bhat and D. S. Bernstein. A topological obstruction to continuous global stabilization of rotational motion and the unwinding phenomenon. Systems and Control Letters, 39(1):63-70, 2000 .

[3] J. D. Biggs and L. Colley. Geometric attitude motion planning for spacecraft with pointing and actuator constraints. Journal of Guidance, Control, and Dynamics, 39(7):1669-1674, 2016.

[4] S. Boyd and L. Vandenberghe. Convex Optimzation, chapter 4. Cambridge University Press, 1st edition, 2004.

[5] J. E. Cochran and J. L. Junkins. Large angle satellite attitude maneuvers. Proceedings of the Flight Mechanics and Estimation Theory Symposium, April 1975.

[6] J. P. Frakes, D. A. Henretty, T. W. Flatley, Markley F. L., J. K. San, and E. G. Lightsey. Sampex science pointing with velocity avoidance. AAS/AIAA Spaceflight Mechanics Meeting, 92-182:949-966, 1992.

[7] E. Frazzoli, M. Dahleh, E. Feron, and R. Kornfeld. A randomized attitude slew planning algorithm for autonomous spacecraft. In Proceedings of the AIAA Guidance, Navigation, and Control Conference and Exhibit, Canada, August 2001.

[8] H. Hablani. Attitude commands avoiding bright objects and maintaining communication with ground station. AIAA Journal of Guidance, Control, and Dynamics, 22(6):759-767, 1999.

[9] N. M. Horri, P. Palmer, and M. Roberts. Gain-scheduled inverse optimal satellite attitude control. IEEE Transactions on Aerospace and Electronic Systems, 48(3):2437-2457, 2012.

[10] John E. Hurtado. Interior parameters, exterior parameters, and a cayley-like transform. AIAA Journal of Guidance, Control, and Dynamics, 32(2):653-657, 2009.

[11] C. Hutao, X. Xiaojun, X. Rui, and C. Pingyuan. RHC-based attitude control of spacecraft under geometric constraints. Aircraft Engineering and Aerospace Technology, 83(5):296-305, 2011.

[12] J.L. Junkins and J. D. Turner. Optimal continuous torque attitude maneuvers. Journal of Guidance and Control, 3(3):210-217, 1980. 
[13] Hassan Khalil. Nonlinear Systems, chapter 4 and 14. Prentice Hall, 3rd edition, 2002.

[14] Ki-Seok Kim and Youdan Kim. Robust backstepping control for slew maneuver using nonlinear tracking control. IEEE Transactions on Control Systems Technology, 11(6):822-829, 2003.

[15] Y. Kim and M. Mesbahi. Quadratically constrained spacecraft attitude constrained control via semidefinite programming. IEEE Transactions on Automatic Control, 49(5):731-735, 2004.

[16] Y. Kim, M. Mesbahi, and F. Hadaegh. Dual-spacecraft formation flying in deep space: Optinal collision-free reconfigurations. Journal of Guidance, 26(2):375-379, 2003.

[17] Y. Kim, M. Mesbahi, G. Singh, and F. Hadaegh. On the convex parameterization of spacecraft orientation in presence of constraints and its applications. IEEE Transactions on Aerospace and Electronic Systems, 46(3):1097-1109, 2010.

[18] H. Kjellberg and G. Lightsey. Discretized constrained attitude pathfinding and control for satellites. AIAA Journal of Guidance, Control, and Dynamics, 36(5):1301-1309, 2013.

[19] J. D. Koenig. A novel attitude guidance algorithm for exclusion zone avoidance. In Proceedings of the 2009 IEEE Aerospace conference, pages 1-10, March 2009.

[20] W. H. Kwon and S. Han. Receding Horizon Control, chapter 3. Springer, 1st edition, 2005.

[21] F. Landis Markley, R. Reynolds, F. Liu, and K. Lebsock. Maximum torque and momentum envelopes for reaction-wheel arrays. Journal of Guidance, Control, and Dynamics, 33(5):16061614, 2010.

[22] U. Lee. State-Constrained Rotational and Translational Motion Control With Applications to Monolithic and Distributed Spacecraft. PhD dissertation, University of Washington, 2014.

[23] U. Lee and M. Mesbahi. Spacecraft reorientation in presence of attitude constraints via logarithmic barrier potentials. Proceedings of the American Control Conference, pages 450-455, 2011.

[24] U. Lee and M. Mesbahi. Quaternion based optimal spacecraft reorientation under complex attitude constrained zones. In Proceedings of the AAS Astrodynamics Specialist Conference, volume 150, pages 1995-2010, Hilton Head, South Carolina, August 2013.

[25] U. Lee and M. Mesbahi. Feedback control for spacecraft reorientation under attitude constraints via convex potentials. IEEE Transcactions on Aerospace and Electronic Systems, 50(4):2578-2592, 2014.

[26] Shih-Che Lo and Yon-Ping Chen. Smooth sliding-mode control for spacecraft attitude tracking maneuvers. Journal of Guidance, Control, and Dynamics, 18(6):1345-1349, 1995.

[27] S. R. Marandi and V. J. Modi. A preferred coordinate system and the associated orientation representation in attitude dynamics. Acta Astronautica, 15(11):833-843, 1987.

[28] C. R. McInnes. Large angle slew maneuvers with autonomous sun vector avoidance. AIAA Journal of Guidance, Control, and Dynamics, 17(4):875-877, 1994.

[29] R. E. Mortensen. A globally stable linear attitude regulator. International Journal of Control, 8(3):297-302, 1968. 
[30] R. Murray. Optimiztion-Based Control, chapter 3. California Institute of Technology, 1st edition, 2009.

[31] K. B. Ngo, R. Mahony, and Z .P. Jiang. Integrator backstepping using barrier functions for systems with multiple state constraints. In Proceedings of the 44th IEEE Conference on Decision and Control, and the European Control Conference, pages 8306-8312, Seville, Spain, December 2005.

[32] A. Oppenheim and R. Schafer. Discrete-Time Signal Processing, chapter 2. Prentice Hall, 2nd edition, 1998.

[33] W. Press, S. Teukolsky, W. Vetterling, and B. Flannery. Numerical Recipes: The Art of Scientific Computing, chapter 5. Cambridge University Press, 3rd edition, 2007.

[34] S. V. Salehi and E. P. Ryan. A non-linear feedback attitude regulator. International Journal of Control, 41(1):281-287, 1985.

[35] H. Schaub, M. R. Akella, and J. L. Junkins. Adaptive realization of linear closed-loop tracking dynamics in the presence of large system model errors. Journal of the Astronautical Sciences, 48(4):537-551, 2000.

[36] H. Schaub and J. L. Junkins. Analytical Mechanics of Space Systems, chapter 3 and 4. AIAA Education Series, Reston, VA, 3rd edition, 2014.

[37] H. Schaub and S. Piggott. Speed-constrained three-axes attitude control using kinematic steering. In Proceedings of the AAS Guidance, Navigation and Control Conference, Breckenridge, CO, February 2017.

[38] H. Schaub, R. D. Robinett, and J. L. Junkins. Globally stable feedback laws for near-minimumfuel and near-minimum-time pointing maneuvers for a landmark-tracking spacecraft. Journal of Astronautical Sciences, 44(4):443-466, 1996.

[39] Otto H. Schmitt. A thermionic trigger. Journal of Scientific Instruments, 15(1):24-26, 1937.

[40] J. Sheen and R Bishop. Adaptive nonlinear control of spacecraft. Proceedings of the American Control Conference, pages 2867-2871, 1994.

[41] S. W. Sheppard. Quaternion from rotation matrix. AIAA Journal of Guidance and Control, 1(3):223-224, 1978.

[42] Malcolm D. Shuster. A survey of attitude representations. Journal of the Astronautical Sciences, 41(4):439-517, 1993.

[43] G. Singh, G. Macala, E. Wong, and R. Rasmussen. A constraint monitor algorithm for the cassini spacecraft. Proceedings of the AIAA Guidance, Navigation, and Control Conference, pages 272-282, 1997.

[44] K. Spindler. New methods in on-board attitude control. Advanced in the Astronautical Sciences, 100(2):111-124, 1998.

[45] C. Sun and R. Dai. Spacecraft attitude control under constrained zones via quadratically constrained quadratic programming. AIAA Guidance, Navigation, and Control Conference, AIAA SciTech, 2015. 
[46] S. Tanygin. Fast three-axis constrained attitude pathfinding and visualization using minimum distortion parameterizations. AIAA Journal of Guidance, Control, and Dynamics, 38(12):2324$2336,2015$.

[47] K. Tee, S. Ge, and E. Tay. Barrier lyapunov functions for the control of output-constrained nonlinear systems. Automatica., 45(45):918-927, 2009.

[48] P. Tsiotras. New control laws for the attitude stabilization of rigid bodies. In Proceedings of the IFAC Symposium on Automatic Control in Aerospace, pages 316-321, September 1994.

[49] P. Tsiotras. Stabilization and optimality results for the attitude control problem. Journal of Guidance, Control, and Dynamics, 19(4):772-779, 1996.

[50] S.R. Vadali. Variable-structure control of spacecraft large-angle maneuvers. Journal of Guidance, 9(2):235-239, 1986.

[51] Thomas Freud Wiener. Theoretical Analysis of Gimballess Inertial Reference Equipment Using Delta-Modulated Instruments. Ph.D. dissertation, Department of Aeronautics and Astronautics, Massachusetts Institute of Technology, Cambridge, MA, March 1962.

[52] R. Wisniewski and P. Kulczycki. Slew maneuver control for spacecraft equipped with star camera and reaction wheels. Control Engineering Practice, 13(3):349-356, 2005. 


\section{Appendix A}

\section{Kinematic Identities}

In this Appendix, some kinematic identities used throughout this work are briefly discussed. See Reference [36] for further reading.

\section{A.1 Transport Theorem}

Let $\mathcal{N}$ and $\mathcal{B}$ be two frames with a relative angular velocity of $\boldsymbol{\omega}_{\mathcal{B} / \mathcal{N}}$ and let $\boldsymbol{a}$ be a generic vector; then the derivative of $\boldsymbol{a}$ in the $\mathcal{N}$ frame $(\dot{\boldsymbol{a}})$ can be related to the derivative of $\boldsymbol{a}$ in the $\mathcal{B}$ frame $\left(\boldsymbol{a}^{\prime}\right)$ as

$$
\dot{a}=a^{\prime}+\omega_{\mathcal{B} / \mathcal{N}} \times \boldsymbol{a}
$$

It is important to understand that this is a vectorial equation that can be written in any frame.

\section{A.2 MRPs Identities}

The MRPs kinematic differential equation is given by 36 .

$$
\dot{\boldsymbol{\sigma}}=\frac{1}{4}\left[\left(1-\boldsymbol{\sigma}^{T} \boldsymbol{\sigma}\right)\left[I_{3 \times 3}\right]+2[\tilde{\boldsymbol{\sigma}}]+2 \boldsymbol{\sigma} \boldsymbol{\sigma}^{T}\right] \boldsymbol{\omega}=\frac{1}{4}[B(\boldsymbol{\sigma})] \boldsymbol{\omega}
$$

The product $\boldsymbol{\sigma}^{T} \dot{\boldsymbol{\sigma}}$ is heavily used in Chapters 2 and 4 . It can be computed as

$$
\begin{aligned}
\boldsymbol{\sigma}^{T} \dot{\boldsymbol{\sigma}} & =\frac{1}{4}\left[\boldsymbol{\sigma}^{T}\left[\left(1-\boldsymbol{\sigma}^{T} \boldsymbol{\sigma}\right)\left[I_{3 \times 3}\right]+2[\tilde{\boldsymbol{\sigma}}]+2 \boldsymbol{\sigma} \boldsymbol{\sigma}^{T}\right]\right] \boldsymbol{\omega} \\
& =\frac{1}{4}\left[\left(1-\boldsymbol{\sigma}^{T} \boldsymbol{\sigma}\right) \boldsymbol{\sigma}^{T}+2 \boldsymbol{\sigma}^{T}[\tilde{\boldsymbol{\sigma}}]+2 \boldsymbol{\sigma}^{T}\left(\boldsymbol{\sigma} \boldsymbol{\sigma}^{T}\right)\right] \boldsymbol{\omega}
\end{aligned}
$$


Using the fact that the matrix $[\tilde{\boldsymbol{\sigma}}]$ is skew-symmetric, the product $\boldsymbol{\sigma}^{T}[\tilde{\boldsymbol{\sigma}}]$ is equivalent to

$$
\boldsymbol{\sigma}^{T}[\tilde{\boldsymbol{\sigma}}]=\left([\tilde{\boldsymbol{\sigma}}]^{T} \boldsymbol{\sigma}\right)^{T}=(-[\tilde{\boldsymbol{\sigma}}] \boldsymbol{\sigma})^{T}=-(\boldsymbol{\sigma} \times \boldsymbol{\sigma})^{T}=\mathbf{0}
$$

Using this result and the associativity of the matrix product

$$
\boldsymbol{\sigma}^{T} \dot{\boldsymbol{\sigma}}=\frac{1}{4}\left[\left(1-\boldsymbol{\sigma}^{T} \boldsymbol{\sigma}\right) \boldsymbol{\sigma}^{T}+2\left(\boldsymbol{\sigma}^{T} \boldsymbol{\sigma}\right) \boldsymbol{\sigma}^{T}\right] \boldsymbol{\omega}=\frac{1}{4}\left(1+\boldsymbol{\sigma}^{T} \boldsymbol{\sigma}\right)\left(\boldsymbol{\sigma}^{T} \boldsymbol{\omega}\right)
$$

Equivalently

$$
\frac{4 \boldsymbol{\sigma}^{T} \dot{\boldsymbol{\sigma}}}{\left(1+\boldsymbol{\sigma}^{T} \boldsymbol{\sigma}\right)}=\boldsymbol{\sigma}^{T} \boldsymbol{\omega}
$$




\section{Appendix B}

\section{Lyapunov Theory}

In this Appendix, some elements of Lyapunov theory are briefly discussed. See References [13, 36] for further reading.

\section{B.1 Lyapunov Stability}

Let the autonomous system

$$
\dot{\boldsymbol{x}}=\boldsymbol{f}(\boldsymbol{x})
$$

have an equilibrium point at zero: $\boldsymbol{f}(\mathbf{0})=\mathbf{0 .} \boldsymbol{f}: D \subseteq \mathbb{R}^{n} \rightarrow \mathbb{R}^{n}$ is locally Lipschitz.

The equilibrium point $\boldsymbol{x}=0$ is

(1) Stable if, for each $\epsilon>0$, there is $\delta=\delta(\epsilon)>0$ such that

$$
\|\boldsymbol{x}(0)\|<\delta(\epsilon) \Longrightarrow\|\boldsymbol{x}(t)\|<\epsilon, \quad \forall t \geq 0
$$

(2) Unstable, if it is not stable.

(3) Asymptotically stable if it is stable and $\delta$ can be chosen such that

$$
\|\boldsymbol{x}(0)\|<\delta \Longrightarrow \lim _{t \rightarrow \infty} \boldsymbol{x}(t)=0
$$

(4) Globally asymptotically stable if it is asymptotic stable for any initial condition. 


\section{B.2 Lyapunov's Direct Method}

Let $\boldsymbol{x}=\mathbf{0}$ be an equilibrium point for $(\mathrm{B} .1)$ and $D \subseteq \mathbb{R}^{n}$ be a domain containing $\boldsymbol{x}=\mathbf{0}$. Let $V: \mathbb{R}^{n} \rightarrow \mathbb{R}$ be a continuously differentiable function such that

(1) $V(\mathbf{0})=0$ and $V(\boldsymbol{x})>0$ in $D-\{\mathbf{0}\}$.

(2) $V(\boldsymbol{x}) \leq 0 \forall \boldsymbol{x} \in D$.

Then $\boldsymbol{x}=\mathbf{0}$ is stable.

Moreover, if

$$
V(\boldsymbol{x})<0, \quad \forall \boldsymbol{x} \in D-\{\mathbf{0}\}
$$

then $\boldsymbol{x}=\mathbf{0}$ is asymptotically stable.

\section{B.3 Constrained Lyapunov Function}

Let $V$ be a proper Lyapunov function for the stable system B.1 and $C$ be some compact (closed and bounded) set such that $D-C$ is a connected set that includes $\mathbf{0}$. Additionaly,

$$
V(\boldsymbol{y}) \rightarrow \infty, \quad \forall \boldsymbol{y} \in \operatorname{border}\{C\}
$$

where border $\{C\}$ is the complement of the interior of $C[4]$.

Then $\boldsymbol{x}$ remains in the open set $D-C$.

\section{Proof}

Since $V$ is positive-definite and $\dot{V}$ is negative semi-definite, $V(\boldsymbol{x}(t)) \leq V(\boldsymbol{x}(0))$. Thus, if $\boldsymbol{x}(0) \in D-C, V(\boldsymbol{x}(t))$ will be bounded for all $t$. Therefore, $\boldsymbol{x}(t) \notin \operatorname{border}\{C\}, \quad \forall t$. Since $\boldsymbol{x}(t)$ is continous, $\boldsymbol{x}(0) \notin C$ and $C$ is compact, $\boldsymbol{x}(t) \notin C, \forall t$ 